\title{
Krise der Moderne und moderne Demokratie: Eric Voegelins neoklassische Interpretation des westlichen Zivilregimes*
}

Das Werk des politischen Philosophen Eric Voegelin erfährt seit Anfang der 90er-Jahre eine stetig wachsende Aufmerksamkeit, die aber bisher vor allem auf seine Kritik der totalitären Bewegungen des 20. Jahrhunderts beschränkt ist. Vor diesem Hintergrund wird Voegelins allgemeine Perspektive auf die Moderne oft als die rein negative Perspektive einer fundamentalen Kultur- und Ideologiekritik wahrgenommen. Der folgende Beitrag stellt die Frage nach Voegelins Verständnis moderner Demokratie und versucht, ein differenzierteres und vielschichtigeres Bild von Voegelins Perspektive zu zeichnen. Seine Interpretation des ,, westlichen Zivilregimes “ betont vor allem die konstitutive Rolle des ,, artikulierten Individuums “ für das Selbstverständnis moderner westlicher Gesellschaften, sieht dabei dieses genuin moderne Selbstverständnis aber zugleich in einer komplexen, kritischen Kontinuität zum klassischen politischen Denken der westlichen Tradition stehen. Voegelins Demokratieverständnis impliziert damit die These von einer gleichsam „neoklassischen“ politischen Moderne, die neben seine bekannte Kritik der problematischen Aspekte der modernen Entwicklung gestellt werden muss.

Inhalt

1. Einleitung

2. Die Demokratiethematik in Voegelins Frühwerk 477

3. Demokratie und moderner Gnostizismus 480

4. Demokratie im deskriptiven, existenziellen und transzendenten Sinn 486

5. Eine „Philosophie der Demokratie“?

6. Schlussfolgerungen $\quad 495$

$\begin{array}{ll}\text { Literatur } & 497\end{array}$

* Der folgende Aufsatz ist die überarbeitete Fassung eines Vortrages, der im Rahmen des X. Internationalen Symposiums des Eric-Voegelin-Archivs am Geschwister-Scholl-Institut für Politische Wissenschaft der Ludwig-Maximilians-Universität München zum Thema „Zur geistigen Krise der westlichen Welt: Eric Voegelins Kritik der Moderne“ am 8. Dezember 2007 gehalten wurde. Ich danke den Teilnehmern des Symposiums und den Gutachtern der ZPol für die aufschlussreiche Kritik und die interessanten Anregungen. 
Hans-Jörg Sigwart

\section{Einleitung}

Eric Voegelin zählt zu den prominenten Figuren der Gründergeneration der deutschen Politikwissenschaft nach 1945 (Noetzel 1991), und er gilt als einer der bedeutendsten Vertreter einer neoklassisch orientierten politischen Theorie bzw. Philosophie im 20. Jahrhundert. In den letzten Jahren ist ein wieder erwachtes Interesse in der politischen Theoriedebatte an „neoklassischen“"Konzeptionen im Allgemeinen zu verzeichnen (Schaal 2006: 517 f.). Insbesondere die Werke von Hannah Arendt, Michael Oakeshott und Leo Strauss sind hier zu nennen. Aber auch Voegelins Werk wird seit Anfang der 90er-Jahre eine deutlich erhöhte Aufmerksamkeit zuteil, ${ }^{1}$ nachdem er zuvor in Deutschland kaum rezipiert worden war (Opitz 1989). Allerdings ist diese neuere Auseinandersetzung mit seinem Werk bisher weitgehend auf einen bestimmten Aspekt beschränkt. Voegelin wird in der gegenwärtigen politikwissenschaftlichen Rezeption meist in den Kontext des ideologie- und kulturkritischen Diskurses des 20. Jahrhunderts gestellt, in dem es vor dem Hintergrund der Erfahrung der totalitären Regime vor allem um die Frage nach einer kritischen, mitunter auch sehr pessimistischen Auseinandersetzung mit der Moderne insgesamt ging. Insbesondere der allerdings äußerst ambivalente „Erfolg“ seiner These vom ,gnostischen Charakter“ der Moderne, die Voegelin vor allem in seinen Schriften der 50er-Jahre vertreten hat, ${ }^{2}$ hat dazu beigetragen, dass er oft ausschließlich in diesem Zusammenhang wahrgenommen wurde und wird. So interessant und eigenständig Voegelins Beiträge zu einer wissenschaftlichen Kritik der problematischen Aspekte der modernen Entwicklung insbesondere im 20. Jahrhundert sind, ihre allzu einseitige Betonung hat dazu geführt, dass eine Vielzahl konzeptioneller Anregungen und konkreter Studien, die Voegelins Werk außerdem bietet, von einem breiteren politikwissenschaftlichen Diskurs bisher nur sehr eingeschränkt wahrgenommen wurde.

Vor dem Hintergrund dieser einseitigen Rezeption - und zu dieser Einseitigkeit hat Voegelin selbst mit seinen in der Tat äußerst markant und zum Teil bewusst polemisch überspitzt vorgetragenen Krisendiagnosen einiges beigetragen - scheint sich insbesondere auf die Frage nach dem generellen Verhältnis, das Voegelin zur politischen und philosophischen Moderne hatte, für viele Interpreten eine relativ klare Antwort aufzudrängen. Für die meisten Autoren, darunter Kritiker ebenso wie solche Interpreten, die Voegelins Werk eher affirmativ rezipieren, lässt sich dieses Verhältnis auf den einfachen Nenner einer umfassenden Fundamentalkritik bzw. den einer „Revolt against Modernity“ (McAllister 1996) bringen. Liest man Voegelins Arbeiten aus den 50er- und 60er-Jahren, dann erwecken sie in der Tat in vielen Passagen den Eindruck, als habe er „die Neuzeit in Bausch und Bogen verdammen“ wollen, wie es Henning Ottmann im dritten Band seiner Geschichte des politischen Denkens mit Bezug auf Voegelin und Leo Strauss formuliert. „Strauss schreibt eine Verfallsgeschichte des Naturrechts, ohne einen Blick zu werfen auf Kant, Hegel oder Tocqueville. Voegelin wird

1 Vor allem Voegelins Konzept der ,politischen Religionen“ zur Analyse und Kritik totalitärer Massenbewegungen und Regime hat in der neueren Debatte viel Aufmerksamkeit erhalten und wird breit diskutiert (dazu Maier 1996 ff.). Mittlerweile ist auch Voegelins Hauptwerk, Order and History, in deutscher Übersetzung zugänglich (Voegelin 2001 ff.). Vgl. dazu auch Henkel 2008.

2 Dazu Voegelin 1959a: 153-223; 1999; Opitz 1999; Hollweck 1999. 
die Gnosis zum großen Tintenklecks, unter dem die Epoche als ganze verschwindet", so Ottmanns Einschätzung, die ihn zu dem kritischen Einwand führt, dass, wer eine solche „reine Verfallsgeschichte“ erzählen wolle, notwendigerweise „die Errungenschaften dieser Epoche leugnen [muss]: den Fortschritt zum Recht des Menschen, die Erringung eines Maßes an Freiheit und Vielfalt der Lebensformen, das es vor der Neuzeit nicht gegeben hat.“ (Ottmann 2006: 3) Musste Voegelin insbesondere diese politischen Errungenschaften ignorieren oder gar leugnen, weil er eine „reine Verfallsgeschichte“ der Neuzeit und Moderne erzählen wollte? Hat die persönliche Erfahrung des Totalitarismus dazu geführt, dass sich Voegelins Perspektive auf die Neuzeit rein auf ihre problematischen Aspekte konzentrierte und damit zugleich reduzierte?

Diese Interpretation kann, vor allem wenn man sich auf Voegelins Arbeiten aus den 50erJahren konzentriert, auf den ersten Blick viel Plausibilität für sich in Anspruch nehmen. Auf den zweiten Blick stößt sie aber doch auf erhebliche Schwierigkeiten. Es stellt sich zum Beispiel die Frage, ob und in welchem Sinne Voegelins eigene Konzeption einer „Neuen Wissenschaft der Politik“ nicht nur als eine „neoklassische“, sondern in vielerlei Hinsicht auch als eine genuin „moderne“ Konzeption politischer Wissenschaft verstanden werden muss. ${ }^{3}$ Wenn das so ist, stellt sich weiterhin die Frage, ob Voegelins Interpretation von Neuzeit und Moderne sich tatsächlich auf eine Fundamentalkritik beschränkt bzw. überhaupt beschränken kann, ohne dabei in Widersprüche hinsichtlich des eigenen Selbstverständnisses als Wissenschaftler zu führen. In der Tat findet sich bei Voegelin neben der Kritik der problematischen Aspekte, die er in der Entwicklung der modernen Philosophie und Wissenschaft sieht (v. a. der moderne Szientismus, Positivismus und reduktionistische Rationalismus sind hier zu nennen), eine breite konstruktive, für seine eigene Position konstitutive Rezeption genuin moderner Konzeptionen. ${ }^{4}$ Diese Frage müsste im Falle Voegelins im Besonderen, aber auch hinsichtlich „neoklassischer“ Konzeptionen im Allgemeinen genauer diskutiert werden, als das bisher der Fall ist. ${ }^{5}$ Während den „klassischen“ Bezügen der entsprechenden Denker viel und auch differenzierte Aufmerksamkeit zuteil wird, steht die spezifische Bedeutung des Präfix „,neo“ bisher nicht in ähnlich deutlicher Weise im Zentrum

3 Vgl. dazu Voegelins Beschreibung dessen, was er unter dem neoklassischen Projekt einer „Restauration der politischen Wissenschaft" verstand (1959a: 19).

4 In diesem Zusammenhang ist u. a. Voegelins konstruktive Rezeption von Max Scheler, Alfred Schütz, Henri Bergson, William James, aber auch, trotz aller Kritik, von Edmund Husserl und Max Weber zu nennen. Vgl. zu Letzterem auch Sigwart 2007b.

5 Diese Frage ist m. E. für die neoklassische politische Philosophie insgesamt gerade im Hinblick auf mögliche Binnendifferenzierungen sehr interessant und aufschlussreich. Vgl. für einen sich allerdings auf die klassischen Bezüge der Autoren konzentrierenden Versuch einer solchen Binnendifferenzierung Ottmann 2005. 
der Aufmerksamkeit. ${ }^{6}$ Die folgenden Überlegungen sollen einen Beitrag zu dieser breiteren Fragestellung leisten.

Man kann in diesem Zusammenhang mit Bezug auf Voegelin einen zweiten Punkt anführen. Denn nicht nur mit Blick auf die wissenschaftliche, sondern auch auf die politische Moderne - und auf diesen Aspekt des Problems scheint zum Beispiel Ottmanns oben zitierte Bemerkung vor allem abzuzielen - stellt sich Voegelins Position bei genauerem Hinsehen ambivalenter und vielschichtiger dar als es zunächst den Anschein hat. Die These vom einfachen Nenner, auf die sich die Gleichung bzw. die Ungleichung Voegelin-Moderne bringen lasse, stößt auf dieser politischen Ebene vor allem auf Schwierigkeiten, wenn man Voegelins Amerika-Erfahrung berücksichtigt, also die wichtige und konstruktive Rolle, welche die persönliche Erfahrung, aber auch die wissenschaftliche Analyse und Interpretation der modernen demokratischen Gesellschaft der USA in Voegelins intellektueller Biografie spielte. Auf diesen letztgenannten Punkt will ich mich im Folgenden konzentrieren und die Frage stellen, welches Verständnis moderner westlicher Demokratie sich bei Voegelin findet und wie es sich zu seiner Fundamentalkritik der Moderne verhält.

Dass diese Fragestellung einen ,zweiten Blick“ auf Voegelins Werk erfordert, liegt vor allem an der Tatsache, dass Voegelin selbst keine systematisch ausgearbeitete Untersuchung zur Demokratieproblematik vorgelegt hat. Zwar spielt in seiner intellektuellen Biografie die Erfahrung der Emigration in die Vereinigten Staaten eine wichtige Rolle. Trotzdem gehört die Demokratieproblematik nicht zu den großen, prominenten Themen in Voegelins Oeuvre. Das Thema wird nirgends zusammenhängend und systematisch, sondern nur am Rande und an verstreuten Stellen seines Werkes behandelt. Das kann man durchaus überraschend finden, vor allem wenn man berücksichtigt, dass nicht nur die Erfahrung der Emigration und die zwei Jahrzehnte, in denen Voegelin als Professor der Politikwissenschaft in den USA arbeitete, ${ }^{7}$ sondern auch die Situation im Nachkriegsdeutschland der späten 50er- und 60er-Jahre (Voegelins Münchner Zeit) eine Beschäftigung mit dem Thema moderne Demokratie durchaus nahegelegt hätten. Auf den ersten Blick scheint sich hier also wiederum die These von der ,reinen Verfallsgeschichte“ zu bestätigen. Ist die wenig prominente Rolle der Demokratiethematik nicht einfach die logische Konsequenz von, die notwendige Entsprechung zu Voegelins Fundamentalkritik der Moderne? ${ }^{8}$

6 Die Frage wird allerdings auch von anderen Autoren diskutiert, zum Beispiel mit Blick auf Voegelins geisteswissenschaftlich-hermeneutisches Wissenschaftsverständnis von Gebhardt 1989, mit Blick auf die phänomenologischen Implikationen von Voegelins Werk von Weiss (2000) und mit Blick auf die Demokratiefrage von Gutschker 2002: 83-87. Schwelling (2005) konzentriert sich auf die konzeptionellen Anregungen, die Voegelins Werk vor dem Hintergrund des ,cultural turn“ der Politikwissenschaft bietet. Vgl. daneben auch Benhabib 1996, die ihre sehr interessante Interpretation Hannah Arendts genau auf die Frage nach ihrem Verhältnis zur Moderne konzentriert. Das Werk von Leo Strauss wird bei Kauffmann (2000) durch den Vergleich mit John Rawls in einen dezidiert modernen Zusammenhang gestellt.

7 Zu Voegelins Biografie vgl. Sandoz 2000.

8 Vgl. zum Beispiel die in diese Richtung gehende Einschätzung von Manfred Henningsen: „The American story simply did not fit into his [Voegelin's, H. S.] theoretical framework; therefore it was left out.“ (2000: 4). Für eine dezidiert kritische Einschätzung von Voegelins Demokratieverständnis vgl. Faber 1984 und Kiel 1998. 
Zunächst spricht einiges dafür, dass man bei der Deutung solcher thematischen „Lücken“sozusagen bei der Interpretation von Büchern, die ein Autor nicht geschrieben hat - sehr zurückhaltend sein sollte. Das Fehlen einer systematischen Abhandlung zur Demokratieproblematik sagt zunächst einmal noch nichts über Voegelins wissenschaftliche Position zu diesem Thema aus. Andererseits bringt Voegelin selbst in der New Science of Politics gerade ein solches Argument - nämlich die kritische Interpretation einer thematischen und sozusagen „materialen“ Lücke - gegen Max Weber vor, und zwar an einer systematisch äußerst wichtigen Stelle seiner Weber-Kritik (Voegelin 1959a: 77 f.). ${ }^{9}$ Voegelins Art der Argumentation an dieser Stelle macht einen wichtigen Punkt in diesem Zusammenhang deutlich, nämlich dass eine „Lücke“ im Werk eines Denkers nur dann von weiter reichender Bedeutung für die Interpretation ist, wenn der thematischen auch eine konzeptionelle „Lücke“, eine gewisse Reduktion, Einseitigkeit oder Unvollständigkeit der Perspektive oder auch Widersprüche und mangelnde Plausibilität etwa bei der Interpretation historischer, empirischer Phänomene entspricht. Metaphorisch ausgedrückt könnte man sagen: wenn eine solche thematische Lücke gleichsam konzeptionelle Phantomschmerzen im Werk hinterlässt.

In der folgenden Untersuchung wird auch diese Frage diskutiert, zunächst einmal werde ich aber zu zeigen versuchen, dass der Eindruck von einer solchen thematischen Lücke erheblich relativiert werden muss. Das Thema moderne Demokratie spielt bei Voegelin eine zwar nicht auf den ersten Blick ins Auge fallende aber dennoch wichtige Rolle. Ich will, um das zu zeigen, zunächst nach dem Demokratieverständnis in Voegelins Frühwerk und nach der intellektuell-biografischen Bedeutung seiner frühen Auseinandersetzung mit der amerikanischen Gesellschaft fragen, um mich im Anschluss auf die Neue Wissenschaft der Politik zu konzentrieren, die bis heute am weitesten rezipierte Schrift Voegelins, an der die These von der „reinen Verfallsgeschichte“ für gewöhnlich festgemacht wird.

\section{Die Demokratiethematik in Voegelins Frühwerk}

Erweitert man die Perspektive auf Voegelins politisches Denken über die vor allem in der deutschen Rezeption stark ausgeprägte Konzentration auf einige wenige seiner Schriften hinaus, dann ergibt sich auch hinsichtlich seines Demokratieverständnisses ein differenzierteres Bild, insbesondere wenn man Voegelins Frühwerk in die Betrachtung mit einbezieht. Denn hier zeigt sich, dass Voegelin sich bereits in den 20er-Jahren intensiv mit der amerikanischen Gesellschaft und Demokratie beschäftigt hat. Zwei Jahre, von 1924 bis 1926, verbrachte Voegelin als Stipendiat der Rockefeller Foundation in den Vereinigten Staaten. Seine erste Monografie, die Habilitationsschrift Über die Form des amerikanischen Geistes von 1928, ist das wissenschaftliche Ergebnis dieser frühen Auseinandersetzung mit der amerikanischen Gesellschaft. Zwar bietet auch diese Arbeit keine systematisch ausgearbeitete Theorie der Demokratie. Allerdings zeigt die Schrift, dass das wissenschaftliche „Problem“ der modernen amerikanischen Gesellschaft schon früh und von hier aus durchgehend eine zwar nicht im Zentrum der Aufmerksamkeit stehende, aber dennoch wichtige Rolle für Voegelin spielt.

9 Vgl. auch Voegelins Kritik an Rudolf Bultmann, in der er ebenfalls die bewussten ,omissions“ Bultmanns zum Gegenstand der Kritik macht (2000: 156-177). 
Die Bedeutung dieser „Amerika-Erfahrung“ zeigt sich zum Beispiel in der Tatsache, dass Voegelin mit ihr schon in den 20er-Jahren seine wissenschaftliche Perspektive bewusst zu einer vergleichenden Perspektive erweitert und dass dabei die Politik bzw. das Politische als der Gegenstand seiner Forschung deutlicher als bisher ins Zentrum der Aufmerksamkeit rückt (Sigwart 2005: 22-30). Voegelins wissenschaftliche Position erfährt also bereits in den 20er-Jahren jene „Politisierung“ und „Internationalisierung“, die Alfons Söllner (1996) als die entscheidenden Charakteristika der Emigrationserfahrung deutscher Politikwissenschaftler der Gründergeneration beschrieben hat. Sie erfährt darüber hinaus auch bereits in den 20erJahren eine deutliche „Demokratisierung“, insofern, als sich schon in Voegelins frühen Arbeiten nicht nur eine profunde Kenntnis, sondern auch eine klare Sympathie für die westlich-angelsächsische Demokratie zeigt. Besonders deutlich ist das etwa in seiner Interpretation des Sozialwissenschaftlers und Historikers der amerikanischen Arbeiterbewegung John R. Commons, den Voegelin in seiner Habilitationsschrift gleichsam als exemplarischen demokratischen Intellektuellen interpretiert (1928: 172-238). ${ }^{10}$ „The real, authentic America“, schreibt Voegelin später in seinen Autobiographischen Reflexionen über den tiefen Eindruck, den Commons in den 20er-Jahren auf ihn hinterlassen hatte, ,was represented by John R. Commons, who took on for me the shape of a Lincolnesque figure.“ (1989: 30)

Die Sympathie für das amerikanische Modell moderner Demokratie zeigt sich aber auch in anderen Schriften aus den 30er-Jahren, etwa in einem „Nachwort“, das Voegelin 1932 zu der deutschen Übersetzung eines in der westlichen demokratischen Tradition stehenden populärphilosophischen Buchs geschrieben hat. Das Buch erfordere, so Voegelin, für den deutschen Leser einige einführende und erläuternde Worte, „die dem Leser die geistige Situation Westeuropas und Amerikas und im Kontrast dazu seine eigene in den Grundzügen verdeutlichen.“ (1932: 280) Ungewohnt für deutsche Leser sei vor allem die Haltung des Autors, „der zu seinen Lesern als zu Gleichen“ spreche und dabei eine die gesellschaftlich-politische Gleichheit, Solidarität und die Dignität des Einzelnen betonende Idee von Humanität artikuliere, die charakteristisch sei für das Selbstverständnis der westlichen Gesellschaften. Diese „demokratische Haltung des Geistes ist für Deutschland ungewohnt, dessen Humanitätsidee zum Teil einen entschiedenen aristokratischen Zug hat.“ (1932: 281) Was Voegelin von dieser spezifisch deutschen Art der „Geistesaristokratie“ - vor allem politisch - gehalten hat, das ist nicht nur in diesem Text von 1932 sehr deutlich, er artikuliert es auch später, etwa in einem Aufsatz von 1964 mit Bezug auf das klassische deutsche Bildungsideal, das im Vergleich zur westlichen Idee des politisch aktiven und gleichberechtigten „Bürgers“ dezidiert geistesaristokratische und eigentümlich apolitische Züge trage:

„Es ist eine merkwürdige Idee, nach der die Bildung wie eine Art Krem manche würden heute sagen Abschaum - auf der Oberfläche der Nation schwimmt und durch die Tätigkeit ihres Schwimmens an der Oberfläche auf mysteriöse Weise die Ordnung im Innern der Nation erzeugen [...] soll. [...] Dagegen zeichnet sich für uns heute [...] in ihrer Folge das politisch-geistige Vakuum einer Nation ab, in dem sich unerfreuliche Dinge wie der National-

10 Vgl. dazu auch Jürgen Gebhardts Einschätzung, Commons habe für Voegelin ,,programmatische Bedeutung in wissenschaftlicher und persönlicher Hinsicht“ und ,,als Repräsentant der demokratischen Idee Amerikas auf Voegelin einen unauslöschlichen Eindruck“ gemacht. (1995: 288) 
sozialismus ansiedeln können. [...] Keine Politik ist so gefährlich wie die der unpolitischen Bildungsidealisten.“ (1964: 110)

Eine zu dieser spezifisch deutschen, apolitischen „Geistigkeit“" im scharfen Kontrast stehende - und übrigens stark christlich geprägte (Voegelin 1932: 286) - Idee der politischen, gesellschaftlichen und auch intellektuellen Gleichheit und eine sich damit verbindende aktive politische Bürgerkultur sind zwei wesentliche Elemente von Voegelins früher Amerika-Erfahrung, die sein wissenschaftliches Demokratieverständnis nachhaltig prägen, bzw. die eine Seite seines Demokratieverständnisses. Denn die angeführten Zitate machen einen weiteren wichtigen Aspekt von Voegelins früher Perspektive deutlich. Er betont in seinen Frühschriften durchgehend die Besonderheit und Verschiedenheit amerikanischer Politik und Geistesgeschichte im Vergleich zur damaligen Situation in Zentraleuropa. Dieser Befund von der Eigentümlichkeit amerikanischer Politik bildet die Folie, vor der Voegelin in den 30er-Jahren die zentraleuropäischen Probleme der Zeit interpretiert. Die amerikanische Demokratie dient dabei immer wieder als Vergleichsgröße, vor deren Hintergrund die Besonderheiten der zentraleuropäischen Situation herausgearbeitet werden.

Besonders deutlich ist das in seinen Studien zum „österreichischen Staatsproblem“ in den 30er-Jahren. Voegelins äußerst skeptische Einschätzung der österreichischen Demokratie vor 1934 beruht vor allem auf der These, dass ihr im Vergleich zur amerikanischen Demokratie die entscheidenden politisch-kulturellen Grundlagen fehlen. Denn zum einen sei Österreich keine moderne „Nation“ mit einer politisch aktiven Bevölkerung, sondern sei erst im Begriff sich langsam zu einer „Nation“ in diesem Sinne zu entwickeln. Und zum anderen vollziehe sich diese überhaupt erst angestoßene Entwicklung hin zu einer ,politischen Nation“ unter der Dominanz kollektivistischer politischer Ideen, die mit der Legitimationsbasis westlich-demokratischer Institutionen eigentlich nicht vereinbar seien. Denn die freiheitlichen Institutionen des westlichen Zivilregimes sind lediglich die äußeren Formen, die „Ramifikationen“, wie Voegelin es ausdrückt, eines politisch-kulturellen demokratischen Selbstverständnisses der Gesellschaft insgesamt, in dessen Kern die Idee von der fundamentalen Gleichheit, der Würde und der Fähigkeit des Individuums zur Selbstregierung steht: „the idea of the individual as a reasonable being, having a scope of experience covering his personal problems, having sufficient intelligence to form an opinion on them, and enough realistic will power to advocate and press them." (1939: 4) Die maßgeblichen politischen Bewegungen in Österreich aber gehören für Voegelin einem anderen, dem zentraleuropäischen kollektivistischen Typus moderner Demokratie an, in dem gerade diese Idee der Würde der Person keine Rolle mehr spiele. In den Kategorien der New Science könnte man diesen Befund so ausdrücken: Der nach westlich-demokratischem Vorbild konzipierten österreichischen Verfassung von 1924 fehlen die repräsentativen Grundlagen im „existenziellen“ und im ,transzendenten“ Sinn. Darauf werde ich noch zurückkommen.

Voegelins frühes Verständnis von moderner Demokratie ist vor dem Hintergrund dieser amerikanisch-zentraleuropäisch vergleichenden Perspektive zu sehen. Beide, sowohl das „westliche Zivilregime“ - wie Voegelin später mit einer Übersetzung von „civil government“ die angelsächsischen Demokratien bezeichnen wird (1959b: 295) - als auch die kollektivistischen, autoritären, aber breite gesellschaftliche Schichten mobilisierenden politischen Bewegungen in Zentraleuropa, waren für Voegelin genuin „,modern-demokratische“ Phänomene. 
Seine frühe Verwendung des Demokratiebegriffs schwankt daher in seinen Implikationen zwischen diesen beiden Varianten, auch was die Frage der „Bewertung“ betrifft. Einerseits zeigt sich immer wieder eine klare Sympathie für die angelsächsische Variante moderner Demokratie - und dabei hebt Voegelin neben der aktiven Bürgerkultur vor allem den besonderen Status, die besondere „Dignität“ hervor, die in der angelsächsischen Tradition die einzelne Person gegenüber der Gesellschaft als ganzer habe. Andererseits beurteilt Voegelin die Chancen für eine Demokratisierung im westlichen Sinne der zentraleuropäischen Gesellschaften meistens sehr skeptisch, ohne diese Tatsache allerdings deutlich zu bewerten. Insgesamt übt sich Voegelin in dieser Frage - wie generell in normativen Fragen - in seinen Frühschriften eher in weberianischer Zurückhaltung und Neutralität.

Diese frühen vergleichenden Studien Voegelins zur Demokratieproblematik spielen für seine Perspektive in den 50er- und 60er-Jahren eine wichtige Rolle. Auch hier steht das Thema nicht im Zentrum der Aufmerksamkeit, aber es hat als immer präsente „Vergleichsgröße“", an der die nun deutlich kritisch beurteilten Besonderheiten vor allem der zentraleuropäischen Entwicklung verdeutlicht werden, eine wichtige Funktion. Hinzu kommt aber jetzt die Frage einer Kritik der Neuzeit und Moderne insgesamt; diese Frage rückt spätestens Ende der 40er-Jahre ins Zentrum von Voegelins Aufmerksamkeit. Und damit ändert sich in bestimmten Punkten auch seine Perspektive auf die westlichen Demokratien. Ich wende mich damit der Neuen Wissenschaft der Politik zu.

\section{Demokratie und moderner Gnostizismus}

Die New Science of Politics ist nicht nur die bekannteste und die bis heute am meisten rezipierte von Voegelins Arbeiten, sie steht auch stellvertretend für eine bestimmte Phase in Voegelins intellektueller Biografie, nämlich für die 50er- und frühen 60er-Jahre. In dieser Phase scheint die These von der modernen Gnosis - die Voegelin selbst später deutlich relativiert hat ${ }^{11}$ - in der Tat eines der zentralen Probleme zu sein, mit denen sich Voegelin beschäftigt. Das entscheidende Charakteristikum der Moderne sei, so im wesentlichen Voegelins Gnosis-These, die Dominanz von in Kontinuität zur antiken Gnosis, zu mittelalterlichen Sektenbewegungen und zum frühneuzeitlichen protestantischen Puritanismus stehenden intellektuellen und politischen Strömungen, die sich durch absolute Gewissheitsund Machbarkeitsvorstellungen und durch die „Immanentisierung“ von ursprünglich

11 Vgl. dazu Opitz 1999: 31-34, der darauf aufmerksam macht, dass sich in Voegelins Arbeiten seit den 60erJahren „die Gnosis-Problematik allmählich wieder aus dem Zentrum seiner Publikationen entfernte“ und der auf verschiedene Stellen in Voegelins späteren Texten hinweist, an denen sich einige die ursprüngliche Gnosis-These in gewissem Sinne relativierende Bemerkungen finden. Allerdings beziehen sich diese Bemerkungen nicht so sehr auf die Frage nach der Beurteilung der Moderne insgesamt, sondern hauptsächlich auf Fragen der Plausibilität der historischen Parallele zwischen antiker und ,moderner Gnosis“, die Voegelin durch neuere Forschungen zu apokalyptischen und neoplatonischen Strömungen zum Teil infrage gestellt sah. Opitz kommt daher zu dem Fazit, dass sich Voegelin auch in seinen späteren Arbeiten weniger mit der „,helle(n) Seite“ der westlichen Moderne als „Weg zu repräsentativer Demokratie, zur Gewaltenteilung und zu den Menschenrechten“ beschäftigt, sondern sich, allerdings ohne diese helle Seite zu bestreiten, vor allem auf die Erklärung ihrer „,dunklen Seiten“ und Gefahren konzentriert habe (1999: 34). 
transzendenten Erlösungsideen auszeichneten. Die totalitären Regime des 20. Jahrhunderts deutet Voegelin in diesem Zusammenhang als die letzte, ungehemmte Konsequenz solcher spezifisch modernen gnostischen Selbsterlösungsideen. Vor dem Hintergrund dieser These vertritt Voegelin in den 50er-Jahren eine kulturkritische Position, aus der heraus er wichtige Bestandteile des modernen Selbstverständnisses insgesamt mit verantwortlich macht für den „Zivilisationsbruch“, den er, ähnlich etwa wie Hannah Arendt, ${ }^{12}$ in den totalitären Massenbewegungen des 20. Jahrhunderts erkannte. Gnostische Motive und Ideen sah Voegelin demnach nicht nur in Faschismus, Kommunismus und Nationalsozialismus, sondern auch in Bewegungen „,von der Art des Progressivismus, des Positivismus, des Marxismus, der Psychoanalyse" (1960: 5) und auch in den Werken klassischer moderner Philosophen, etwa bei Hegel, Marx, Nietzsche und Heidegger (Voegelin 1959c) vertreten. Der Totalitarismus erscheint vor diesem Hintergrund als Konsequenz einer geistesgeschichtlichen Entwicklung innerhalb des Westens, deren Wurzeln bis in die Spätantike zurückreichen und die insbesondere das politische Selbstverständnis der Moderne entscheidend geprägt habe.

Voegelin glaubt offenbar, mit dem Begriff der „Gnosis“ die Entdeckung gemacht zu haben, mit der die Frage beantwortet werden kann, die im Zentrum seiner wissenschaftlichen Bemühungen seit den späten 30er-Jahren gestanden hatte: die Frage nach der Möglichkeit einer kritischen wissenschaftlichen Auseinandersetzung mit den Totalitarismen des 20. Jahrhunderts. Zuvor standen diese Frage und ihre vielfältigen Implikationen im Zentrum von Voegelins Aufmerksamkeit, jetzt rückt eine spezifische Antwort ganz in den Vordergrund; und damit ändert sich auch der Stil seiner Arbeiten. Das ist insbesondere in jenen Schriften deutlich zu erkennen, die in dem Bewusstsein geschrieben sind, dass sie von einer breiteren Öffentlichkeit wahrgenommen werden würden. Das gilt für die New Science, die auf die „Walgreen Lectures“ zurückgehen, die Voegelin 1951 in Chicago gehalten hat, ebenso wie für die Münchner Antrittsvorlesung Wissenschaft, Politik und Gnosis (1959c) und die Vorlesung zum Thema Hitler und die Deutschen (2006), in der Voegelin die deutsche Nachkriegsgesellschaft nicht nur mit einem äußerst kritischen Befund, sondern auch mit einer Reihe bewusst gesetzter Provokationen konfrontiert. Man ginge zu weit, wenn man diese Arbeiten als politisch-intellektuelle Kampfschriften bezeichnen würde, aber mir scheint zumindest klar erkennbar zu sein, dass Voegelin in den genannten Schriften neben der differenzierten Darstellung politikwissenschaftlicher Probleme die bewusste Provokation sehr wichtig war.

Damit verbindet sich eine zweite Eigentümlichkeit der New Science, die bei ihrer Interpretation zu berücksichtigen ist. Besonders in den Passagen, in denen Voegelin moderne und unmittelbar zeitgenössische Probleme behandelt, scheint seine Argumentation nur zum Teil von einer „neoklassischen“ Perspektive im eigentlichen Sinn des Wortes, daneben aber auch

12 Für eine prägnante Darstellung von Hannah Arendts diesbezüglicher Position vgl. Barley 1990: 25 ff., der unter anderem darauf hinweist, dass auch für Arendt der Totalitarismus ein genuin modernes Phänomen sei und „bestimmten Tendenzen unseres Zeitalters“ entspreche (27). Zu Arendts allgemeiner Konzeption von Kulturkritik, in der übrigens ähnlich wie bei Voegelin die amerikanische Demokratie die Rolle des positiven Gegenbilds zur europäischen Entwicklung spielt, vgl. Barnouw 2003; Bluhm 2003 und Geisen (2003) sowie den interessanten Beitrag von Thaa 2005, der Arendts Position mit der Kulturkritik Max Webers und der der Frankfurter Schule vergleicht und vor diesem Hintergrund nach den jeweiligen demokratietheoretischen Implikationen fragt. 
deutlich von einem dezidierten politischen Realismus geprägt zu sein. Es finden sich zahlreiche klassisch ,realistische“ Topoi in der Neuen Wissenschaft, ${ }^{13}$ und auch die nicht selten bewusst provokative und polemische Art und Weise, wie Voegelin sie hier verwendet, könnte man als Spezifikum eben dieser Tradition verstehen. ${ }^{14}$ Neben dem Motiv, eine wissenschaftliche, differenzierte Perspektive zu entwickeln, steht hier das Motiv der bewussten Provokation eines öffentlichen Diskurses in den westlichen Gesellschaften, der aus Voegelins Sicht durch eine anti-realistische, ,gesinnungsethische“ Schieflage geprägt ist, die den politischexistenziellen Realitäten und Notwendigkeiten gegenüber blind oder zumindest kurzsichtig mache. ${ }^{15}$ Die New Science ist sicherlich nicht nur, aber sie ist auch als eine solche in der realistischen Tradition stehende Polemik und Provokation geschrieben, die sich gegen einen gesinnungsethischen Idealismus und „Provinzialismus“ richtet, durch die Voegelin die öffentliche Debatte dominiert sah (1959a: 79). Die Neue Wissenschaft der Politik knüpft daher nicht nur an die „neoklassische“, sondern auch an die „realistische“ Tradition an und lässt in manchen Passagen, besonders im Stil der Argumentation, eher an den Principe, den Leviathan und Webers politische Schriften denken als an die Politeia oder die Nikomachische Ethik. Diese Besonderheiten des Textes spielen, wie gleich noch deutlicher werden wird, auch im Hinblick auf die „Demokratie“-Frage eine Rolle und sollten daher als Hintergrund mit berücksichtigt werden.

Wie steht es nun mit der Interpretation des westlichen Zivilregimes in der New Science of Politics? Im letzten Absatz der Neuen Wissenschaft spricht Voegelin von einem „Hoffnungsstrahl“", den er in die ansonsten durchgehend problematische aktuelle Situation, insbesondere hinsichtlich der Konstellationen in der internationalen Politik, fallen sieht: Denn

13 Zu nennen ist hier etwa: die Bedeutung, die Voegelin der „existenziellen“ Problematik der Durchsetzungsfähigkeit von Regimen nach innen und des Überlebens politischer Gesellschaften und ihrer relativen Stabilität und Durchsetzungsfähigkeit auf internationaler Ebene zuschreibt; die Betonung der Rolle politisch und intellektuell herrschender Minderheiten; die Ablehnung nicht nur einer geschichtsteleologischen Perspektive, sondern auch jeglicher Form von Progressivismus und Reformismus und dagegen die Betonung des unentrinnbaren Zyklus der Begründung, des Gedeihens und des anschließenden Verfalls politischer Ordnungen; das Insistieren auf einer realistischen Perspektive, welche die Notwendigkeiten der existenziellen politischen Problematik und die engen Beschränkungen des Möglichen durch die Wechselfälle und Zufälligkeiten in einer letztlich als irrational, chaotisch, kontingent erfahrenen Welt der politischen, menschlichen Dinge ins Zentrum der Aufmerksamkeit rückt. (Vgl. z. B. Voegelin 1959a: 55, 77, 201, 229-235). Vgl. schließlich die Passagen zu Thomas Hobbes, die dann allerdings auch zeigen, dass mit dieser Art politischen Realismus für Voegelin gerade nicht das letzte Wort der politischen Theorie gesprochen ist (Voegelin 1959a: 211-224 und 245-253). Umso bemerkenswerter ist es aber, dass er dieser Hobbes'schen „realistischen“ Fragestellung - dem, wie Voegelin schreibt, ,theoretisch elementaren Aspekt unseres Themas“ (Voegelin 1959a: 55) - weite Passagen der New Science widmet.

14 Quentin Skinner etwa betont in seiner Interpretation Machiavellis, dass ,die originellsten und schöpferischsten Aspekte seiner politischen Anschauungen am besten als eine Reihe polemischer - und manchmal satirischer - Reaktionen auf das System der humanistischen Glaubenssätze“ seiner Zeit zu verstehen seien, die allerdings auch seine eigene Perspektive geprägt hätten. Machiavelli selbst müsse daher ungeachtet seiner realistischen Polemik als Exponent „einer ausgeprägten humanistischen Tradition des klassischen Republikanismus“" gelesen werden (1988: 7).

15 Vgl. etwa Voegelin 1959a: 233, wo er implizit die klassisch weberianische Position einer politischen Verantwortungsethik vertritt. 
„die amerikanischen und englischen Demokratien, die in ihren Institutionen die Wahrheit der Seele am stärksten repräsentieren, sind gleichzeitig auch existenziell die stärksten Mächte.“ Die westlichen Zivilregime sind die Kräfte des Widerstands gegen die ,gnostische Korruption“ der Zeit, sind die „Kräfte der Zivilisation“, wie Voegelin es hier formuliert (1959a: 259). Aber inwiefern stehen sich Gnostizismus und westliche Demokratie als Gegensätze gegenüber? Die unmittelbar vorangehenden Abschnitte - und viele weitere Stellen in den vorhergehenden Kapiteln (1959a: 226, 236, 238, 253) - zeigen, dass auch die westlichen Demokratien von der Bewegung der modernen Gnosis beeinflusst und geprägt sind. Voegelins Krisendiagnose bezieht sich durchaus auch auf die westlichen demokratischen Gesellschaften, allerdings in differenzierter Weise. Die ,westliche Gesellschaft als Ganzes“ wird von Voegelin als eine ,vielschichtige Zivilisation“ charakterisiert, ,in welcher die amerikanische und englische Demokratie die älteste, am festesten konsolidierte Schicht kultureller Tradition darstellt, während der deutsche Bereich ihre fortschrittlichste, modernste Schicht bildet.“ (1959a: 259) Auch die angelsächsische „Schicht“ - oder man könnte sagen: Phase der Moderne (denn der Zeitfaktor spielt hier offenbar eine wichtige Rolle) - ist aus Voegelins Sicht von gnostischer Modernität beeinflusst, aber im Vergleich zu ihren fortschrittlicheren Phasen nur in eingeschränkter, durch die weiterhin lebendigen Traditionen klassischen westlichen Denkens gleichsam eingehegter Weise. Besonders für die angelsächsischen Demokratien gilt daher, dass die zeitgenössische ,westliche Gesellschaft nicht durch und durch modern ist, sondern dass die Modernität etwas in ihr Gewachsenes, ihrer klassischen und christlichen Tradition Entgegengesetztes ist.“(Voegelin 1959a: 242)

In den angelsächsischen Gesellschaften, so offenbar Voegelins Argument, steht dem Neuen das Alte, die weiterhin einflussreiche klassische Tradition entgegen. Sind also die westlichen Demokratien nur deshalb Elemente des Widerstands gegen den „Gnostizismus“ und also gegen die modernen Massenideologien, weil und insofern sie nicht vollständig „moderne“ Phänomene sind? Muss das westliche Zivilregime demnach letztlich als „Anachronismus“ innerhalb der Neuzeit verstanden werden, das im Voegelin'schen Narrativ einer „reinen Verfallsgeschichte“ gleichsam die Rolle des „Katechon“ spielt, ${ }^{16}$ die Rolle des retardierenden, aufhaltenden Moments, das aber gerade deshalb innerhalb der Gesamtbewegung der Moderne nur als Fremdkörper verstanden werden kann? Die amerikanische Gesellschaft erschiene vor diesem Hintergrund zwar als genuin moderne Gesellschaft, als solche aber ihrerseits als Erscheinungsform der modernen Krise; ihr ,paradigmatischer Wert“ hingegen läge darin, dass sie eine lediglich eingeschränkt moderne Gesellschaft ist, das Phänomen einer „restrained modernity“, während der Totalitarismus als „,modernity without restraint“, als „hemmungslose Modernität“ beschrieben wird (Voegelin 1959a: 259).

Das ist das Bild, das die Lektüre der einschlägigen Kapitel der Neuen Wissenschaft zunächst hinterlässt. Aber es bleibt einigermaßen vage, da Voegelin lediglich eine sehr grobe Skizze dieser Zusammenhänge gibt. In einem Brief von 1954 an einen Harvard-Studenten, der um weitere Klärung dieser groben Skizze gebeten hatte, gibt Voegelin einige weitere Hinweise. „What particular institutions, ideas, and men in the American tradition at its

16 Der ursprünglich biblische Begriff des „Katechon“ (des Aufhalters) spielt vor allem in der Kulturkritik Carl Schmitts, insbesondere in seinen Schriften nach 1945, eine wichtige Rolle. Vgl. dazu Leutzsch 1994: 187-191. 
founding and in the contemporary period do I see as characterizing a resistance to gnosticism“, fasst Voegelin die an ihn gestellte Frage zusammen - eine Frage, die aus verschiedenen Gründen schwer zu beantworten sei, unter anderem aus dem folgenden: „[T]he resistance is not a positive action, but negatively is characterized by the absence of gnostic movements and ideas of the more virulent type. Hence, the best brief answer will be a listing of the important bulwarks that have remained uncorroded by gnostic tendencies“. (2007: 212) Das oben skizzierte Bild scheint sich auch hier zu bestätigen: Auch in der amerikanischen Gesellschaft finden sich gnostische Einflüsse, aber ihre Auswirkungen bleiben beschränkt. Die normative Bedeutung der westlichen Demokratie ist eine rein „negative“, liegt in ihrem nicht-gnostischen Charakter.

Voegelins folgende Liste der ,,more important bulwarks“ allerdings will nicht in dieses Bild hinein passen. Die Liste nennt die folgenden Punkte: 1. die föderale Struktur und politische Kultur der Vereinigten Staaten, die ein ,centralized government and its control by an ideological hierarchy“, verhindere, ,which is characteristic of all serious gnostic movements from Boniface VIII. to Karl Marx" (2007: 212); 2. eine im Kern auf die Unverletzlichkeit der einzelnen Person konzentrierte „,politische Theologie“ (2007: 12), die im Zentrum des amerikanisch-demokratischen Selbstverständnisses stehe; 3. die Stabilität und Funktionsfähigkeit der parlamentarischen Institutionen, die auf einen unideologischen, grundsätzlich kooperativen Politikstil zurückzuführen sei; 4. die Besonderheiten amerikanischer Rechtstradition - mit einem expliziten Verweis auf Tocqueville. Und schließlich läuft die Liste 5. auf ein allerdings sehr ambivalentes Lob der politischen Anthropologie hinaus, die sich im amerikanischen Selbstverständnis spiegele. Einerseits zu Extremen neigend, andererseits im Ganzen die Extreme gegeneinander ausbalancierend, sei dieses eigentümlich amerikanische Menschenbild, wie es etwa bei Jefferson, Hamilton und Madison zum Ausdruck komme, „probably the saving feature.“ (2007: 213$)^{17}$

Diese Liste relativiert das Bild vom rein negativen Charakter westlicher Demokratie deutlich. Die genannten, den „Gnostizismus“ beschränkenden Faktoren - Föderalismus, Parlamentarismus, Rechtsförmigkeit und eine den besonderen Status des Einzelnen betonende „politische Theologie“"18 - können wohl nicht ohne erhebliche Probleme als genuin nichtoder gar „anti-moderne“ Phänomene beschrieben werden. Dass das moderne „westliche Zivilregime" dagegen in der Tat einen eigenständigen ,paradigmatischen Wert" habe, der über einen rein ,,negativen“ Aspekt hinausgeht, macht Voegelin bereits im Vorwort zur deutschen Ausgabe der Neuen Wissenschaft deutlich:

17 Vgl. auch Voegelin 1959b: 295, wo Voegelin als wesentliche Funktionsbedingungen des Zivilregimes außerdem die Prinzipien des ,limited government“, der Gewissens-, Wissenschafts- und Religionsfreiheit sowie der wehrhaften Demokratie betont.

18 An einer anderen Stelle desselben Briefs verwendet Voegelin den lateinischen Terminus ,theologia civilis“ (2007: 213). Der Begriff der ,politischen Theologie“ ist hier insofern irreführend, als Voegelin in der Sache nicht so sehr an Carl Schmitt anknüpft, sondern eher auf solche Zusammenhänge hinweisen will, die im Anschluss an Robert Bellahs klassische Studie im Allgemeinen mit dem Begriff der „Zivilreligion“ beschrieben werden. Der Begriff der Zivilreligion würde daher der Sache nach hier besser passen. Für eine sich in diesem Sinn an Voegelin anschließende Konzeptualisierung von „Zivilreligion“ vgl. Gebhardt 2004. 
„Aus der mediterranen Vergangenheit sind der westlichen Welt zwei große Substanzmassen der Ordnung überliefert worden: 1. die klassische Politik und 2. die jüdisch-christliche Ontologie von Mensch, Gesellschaft und Geschichte. Das angelsächsische Zivilregime ist der prinzipiell und weltgeschichtlich bedeutsame Versuch, diese beiden Massen von Ordnungssubstanz adäquat zu institutionalisieren - zuerst für den Nationalstaat alten Stils und heute für die moderne Industriegesellschaft. Das Zivilregime überragt an nomothetischer Kunst und praktischem Erfolg alle anderen Verfassungsexperimente der Neuzeit: für die nachmittelalterlichen, modernen Gesellschaften sind seine Prinzipien an paradigmatischem Wert den Modellkonstruktionen eines Platon und Aristoteles für die Polis zu vergleichen.“"(1959a: 16)

Das westliche Zivilregime steht für Voegelin offensichtlich einerseits für einen ,,konservativen“ Aspekt der „American Tradition“, für ihre Verwurzelung in der griechisch-jüdischchristlichen Tradition westlichen politischen Denkens. Aber diesem „konservativen“ entspricht andererseits ein progressiver, genuin „moderner“ Aspekt, denn das Zivilregime „artikuliert“ die „Ordnungssubstanz“ dieser Tradition für die modernen Formen von Nation und Industriegesellschaft. Gerade in dieser Artikulation ist das Zivilregime ein genuin neuzeitliches, modernes soziopolitisches und geistiges Phänomen, eben der ,prinzipiell und weltgeschichtlich bedeutsame" Versuch, die politischen, nomothetischen und praktischen Implikationen der Tradition vor dem Hintergrund der Moderne konsequent herauszuarbeiten - man könnte mit einem Voegelin'schen Terminus auch sagen: zu ,differenzieren“. ${ }^{19}$ Das Zivilregime als genuin „neuzeitliches Verfassungsexperiment“ hat sein Fundament in einer klaren Kontinuität zur Klassik westlichen politischen Denkens, zeichnet sich zugleich aber - vor allem in seinen politisch-institutionellen Konsequenzen - durch eine spezifische „Modernitäat $t^{\prime \prime}$ aus (Voegelin 1959a: 242, 255, 259). Hier wird eine grundsätzlich andere Perspektive auf die Moderne erkennbar, als es Voegelins Krisendiagnosen zunächst nahelegen: In der Interpretation des Zivilregimes geht es nicht mehr um einen einfachen, klaren, an keiner Stelle vermittelten Gegensatz zwischen Klassik und Neuzeit, sondern es geht gerade um die Frage einer genuinen Verbindung von klassischer Tradition und Modernität - und, wie gleich noch deutlicher werden wird, um die Frage der Balance von Kontinuität und Modernität in diesem Sinn.

19 Mit dem Begriffspaar „compactness“ und „differentiation“ versucht Voegelin in seinen geschichtstheoretischen Arbeiten die Struktur geistiger und soziokultureller Entwicklungsprozesse zu beschreiben, in denen kulturelle Konstellationen, intellektuelle Perspektiven und entsprechende symbolische Artikulationen sich gegenüber zuvor kompakteren Konstellationen und Vorstellungen historisch ausdifferenzieren und damit zu komplexeren Realitätsverständnissen führen. Voegelins Begrifflichkeit betont dabei insofern ein prinzipielles Element der Kontinuität im Verlauf solcher geistig-kulturellen Umbrüche, als die kompakteren Symbolformen in bestimmter Hinsicht auch nach einem solchen historischen Differenzierungsschritt ihre Geltung bzw. Bedeutung behalten. Die ,,kosmische Primärerfahrung“ zum Beispiel, die in der Symbolform des Mythos artikuliert werde, bleibe auch in der klassischen Philosophie etwa Platons und Aristoteles' ein fundamentaler Bestandteil der symbolischen Wirklichkeitsbeschreibung. Dazu exemplarisch Voegelin 2005: 138-145; 1974: 8-11. 


\section{Demokratie im deskriptiven, existenziellen und transzendenten Sinn}

Ein solchermaßen komplizierteres Verhältnis zwischen Zivilregime und Moderne klingt in verschiedenen Passagen der Neuen Wissenschaft an, zum Beispiel im Zusammenhang von Voegelins theoretischer Unterscheidung von Repräsentation im deskriptiven, im existenziellen und im transzendenten Sinn. Insbesondere ein Blick auf dieses theoretische Kernstück der Schrift kann den Sachverhalt noch genauer klären helfen. Voegelin entwickelt im ersten und zweiten Kapitel der Neuen Wissenschaft die These, dass eine politisch-theoretische Betrachtung des Repräsentationsproblems über die gängige ,deskriptive“ Variante des Begriffs, der sich tendenziell auf technisch-institutionelle Aspekte beschränke, hinauskommen müsse (1959a: 55). Nur ein über diese technischen Aspekte hinaus auch soziokulturelle Zusammenhänge integrierendes Verständnis des Repräsentationsbegriffs ermögliche es, die politisch-theoretischen Grundprobleme des Themas zu erfassen. Voegelin hat dabei vor allem zwei „Grundprobleme“ im Blick, nämlich erstens die Frage der „Artikulierung“, d. h. der Konstituierung bzw. Formierung von Gesellschaften als handlungsfähige, nach innen und außen als Akteur auftretende politische Zusammenhänge: die Frage der ,existenziellen Repräsentation“ (Voegelin 1959a: 61 f.). Das Bestehen einer politischen Gesellschaft in dem Sinne, dass sie nach innen und nach außen in ihrer Gesamtheit als Akteur auftreten kann (etwa als die Bundesrepublik Deutschland) hat ihre „Artikulierung“ zur Voraussetzung, und das heißt, dass ein existenzieller Repräsentant (eine Person, eine Institution, eine Bewegung, ein Ensemble von Institutionen, ein politisches System) den Anspruch erhebt, im Namen der Gesellschaft als Ganze zu handeln und dass diesem Anspruch ein gesamtgesellschaftlicher Minimalkonsens darüber entspricht, dass er „Zurecht“ erhoben wird. Dieser Minimalkonsens kann sich auf die Übereinstimmung darüber, dass die Gesellschaft als „Ganzes“ tatsächlich existiert und gegenüber den „Repräsentanten“ auf bloße Fügsamkeit und den Verzicht auf Widerstand beschränken, er kann aber auch, wie wir noch sehen werden, sehr viel weitergehenden Inhalts sein und damit einen wesentlich höheren Grad an „Artikulierung“ der Gesamtgesellschaft erreichen. ${ }^{20}$

Der Begriff der „Artikulierung“ konzentriert sich also auf die Frage, wie die Konstitution von Gesellschaften als politische Gesamtzusammenhänge formal beschrieben werden

20 Voegelins Begriff der existenziellen Repräsentation lehnt sich zum Teil an Carl Schmitts Überlegungen zum Begriff der „politischen Existenz“, vor allem aber eng an Max Webers Legitimitätstheorie an. Die Nähe geht bis in frappierende Ähnlichkeiten in der Begrifflichkeit und der Art der Formulierung hinein. Vgl. etwa Voegelin 1959a: 74 zur Bedeutung persönlichen „Charismas“ in diesem Zusammenhang oder die Wendung vom „regelmäßigen Gehorsam“, die sich deutlich an Webers Definition des Herrschaftsbegriffs anlehnt (Voegelin 1959a: 61). Allerdings geht Voegelin in einem wichtigen Punkt über Weber hinaus, nämlich in der konzeptionellen Betonung des „Ganzen“ der politischen Gesellschaft, auf das jeder existenzielle „Legitimitätsglaube“ bezogen ist (dazu Sigwart 2007a). Vgl. in diesem Zusammenhang auch Pitkin 1967: 44-47, die Voegelins Konzeption existenzieller Repräsentation als eine Variante des „authorization view“ des Repräsentationsproblems interpretiert, der auf die Hobbes'sche Vorstellung von Repräsentation als mehr oder weniger unumschränkte Autorisierung zurückgehe. 
kann. ${ }^{21}$ Darüber hinaus betont Voegelins Repräsentationstheorie aber zweitens das „Grundproblem", dass die Selbstverständnisse solcher gesellschaftlichen Sinn- und Handlungszusammenhänge bzw. -einheiten dazu tendieren, sich selbst als Repräsentanten einer über das bloße Faktum ihrer historischen Existenz hinausgehenden „Wahrheit“ zu verstehen. Der Begriff der „transzendenten Repräsentation“ formuliert also die These, dass jede institutionelle und geistige Ordnung einer politischen Gesellschaft den substanziellen Anspruch impliziert, eine über ihre bloße Existenz hinausgehende Legitimität zu besitzen und dass dieser Anspruch generell universalistische Tendenzen hat (Voegelin 1959a: 83-92). ${ }^{22}$ Die Idee der politischen Legitimität bestimmter institutioneller Arrangements beruht demnach letztlich, so die empirisch gemeinte These, auf dem Anspruch von Gesellschaften, in ihrem politisch-kulturellen Selbstverständnis an von ihnen selbst unabhängig geltende Normen (etwa traditionell: an das „Naturrecht“, oder modern: an die „Würde des Menschen“ oder die Menschenrechte) angebunden zu sein. ${ }^{23}$ Gesellschaften ,repräsentieren“ in ihren Grundprinzipien „Wahrheiten“, die sozusagen über die kulturellen, historischen und geografischen Grenzen der Gesellschaft hinaus als geltend verstanden werden. ${ }^{24}$

Auf diese beiden Varianten des Repräsentationsbegriffs (existenzielle und transzendente Repräsentation) konzentriert sich die Neue Wissenschaft der Politik. Die für die Demokratiethematik wichtigen Implikationen dieser Repräsentationstheorie sind aber zunächst gerade in der Variante des Repräsentationsbegriffs zu suchen, die in der konzeptionellen Anlage der New Science in den Hintergrund rückt: der Repräsentation im „deskriptiven“ Sinn. Denn bei der deskriptiven Repräsentation im Voegelin'schen Sinn handelt es sich um die Beschreibung jener Institutionen und Prozesse, wie sie für moderne westliche Gesellschaften und insbesondere für die angelsächsischen Zivilregime charakteristisch sind. Zwar beschränkt sich dieser Begriff zunächst auf rein äußerliche Institutionen und Prozesse. Gleichzeitig hat die Idee „deskriptiver Repräsentation“ aber tieferliegende Voraussetzungen und Implikationen, die ebenfalls eigentümlich sind für westliche demokratische Gesellschaften.

21 Die Frage nach der „Einheit“ des Staates in diesem Sinne spielt auch in der Staatsrechtslehre der 20erund 30er-Jahre eine zentrale Rolle (Llanque 1995). Die Staatsrechtslehre der Zeit gehört zu den wissenschaftlichen Diskussionszusammenhängen, in denen sich Voegelin in den 20er- und frühen 30er-Jahren vor allem bewegte (Henkel 2003).

22 Diese universalistische Tendenz transzendenter Repräsentationsverständnisse hat Voegelin sehr ambivalent beschrieben, als ein empirisch und funktional offenbar unvermeidliches Element politisch-kultureller Selbstverständnisse, das aber insbesondere in ihren politischen, potenziell konfliktträchtigen und imperialistischen Konsequenzen auch problematische Implikationen habe (z. B. 1959a: 89 f.).

23 Der Begriff der „transzendenten Repräsentation“ muss insofern in einem weiteren als im vielleicht zunächst naheliegenden Sinn verstanden werden, als damit nicht nur und nicht notwendigerweise ,weltjenseitige“, ,religiöse“ Legitimitätsideen angesprochen sind. Dazu Aron Gurwitschs entsprechende „,phänomenologische" Interpretation des Begriffs, die er in der Korrespondenz mit Voegelin und Alfred Schütz über die Neue Wissenschaft skizziert (Voegelin/Schütz/Strauss/Gurwitsch 1993: 133-143).

24 Die Spannung zwischen universeller und relativistisch-kontextualistischer Geltung bzw. Begründung soziopolitischer Grundprinzipien und Selbstverständnisse, auf die Voegelins Begriff der „transzendenten Repräsentation" analytisch abzielt, spielt unter anderem in der aktuellen Diskussion um die Geltung der Idee der Menschenrechte eine zentrale Rolle. Vgl. dazu die prägnante Darstellung bei Menke/Pollmann 2007: 71-128. 
Durch die Differenzierung des Repräsentationsbegriffs und die Konzentration auf seine „existenziellen“ und ,transzendenten“"Implikationen verschiebt Voegelin also zwar zunächst den Fokus weg von der spezifischen institutionellen und Legitimationsproblematik moderner westlicher Gesellschaften, die gängigerweise mit dem Begriff verbunden wird. Und der Stil, in dem Voegelin diese Verschiebung begründet, zeigt, dass auch dieses Argument nicht nur, aber auch eine bewusst gesetzte Provokation darstellt, die einen ,realistischen“ Akzent zu setzen und vor allem wissenschaftliche Tabus und Selbstverständlichkeiten herauszufordern versucht. ${ }^{25}$ Voegelin optiert damit aber nicht für einen anti-demokratischen Repräsentationsbegriff - diesen Verdacht hegt etwa Hans Kelsen (2004: 45, 50 f., 53) in seiner 1954 geschriebenen Erwiderung auf die New Science - sondern er will die Perspektive auf den weiteren ,theoretischen Rahmen“ verschieben, in dem auch die demokratische Repräsentationsproblematik zu betrachten sei, und er versucht dadurch vor allem , die sehr speziellen historischen Bedingungen verständlich [zu machen], unter denen sich die gemeinhin als solche bezeichneten repräsentativen Institutionen [im deskriptiven Sinn, H. S.] entwickeln können. Es wurde bereits angedeutet, dass sie nur in der griechisch-römischen und in der westlichen Kultur auftreten; und die Bedingung ihrer Entwicklung wurde vorläufig als der Prozess formuliert, in dem sich das Individuum zur vertretbaren Einheit artikuliert." (Voegelin 1959a: 78)

,[T] he articulation of the individual as a representable unit," heißt es im englischen Original (Voegelin 1987: 50). Diese Formel, die Voegelin in der New Science an mehreren Stellen verwendet ${ }^{26}$ und die bereits in seinem Frühwerk zu finden ist (1928: 180), macht für ihn offenbar den Kern des Selbstverständnisses moderner demokratischer Gesellschaften aus, und ein Blick auf den historischen Prozess der „Artikulierung des Individuums“ zu einer repräsentierbaren Einheit, wie Voegelin ihn skizziert, zeigt, dass dieses Selbstverständnis eigentümliche Implikationen auch für die Repräsentationsproblematik im „existenziellen“

25 Dass Voegelin die Provokation vor allem in diesem Punkt gelungen ist, zeigt ein Blick in die ausführliche zunächst unveröffentlicht gebliebene Kritik, die Hans Kelsen 1954 gegen die New Science formuliert hat: „What is $[. .$.$] the reason for presenting the definition of a representative form of government as a govern-$ ment elected by the people on the basis of universal suffrage as merely elemental, to be replaced by a more appropriate one, the ,existential' definition? The allegedly elemental definition of representation is the definition of a certain type of representation: representation of a community organized by a democratic constitution. It is of importance to note that the type of representation which the new science of politics depreciates as elemental is that sort of representation which is the essence of democracy." (Kelsen 2004: 36 f.)

26 Vgl. neben den im Folgenden genannten auch die interessante Stelle in der Neuen Wissenschaft (1959a: 104 f.), wo Voegelin die Formel mit Bezug auf die athenische Polis zur Zeit von Marathon und der Tragödie verwendet: „Hier hatte sich für eine goldene Stunde der Geschichte das Wunder einer bis hinunter zum Einzelbürger als repräsentierbarer Einheit artikulierten politischen Gesellschaft ereignet - das Wunder einer Generation, welche die Verantwortung, die Wahrheit der Seele zu repräsentieren, individuell erfahren hatte und dieser Erfahrung durch die Tragödie als öffentlichem Kult Ausdruck verlieh.“ An dieser Stelle spricht Voegelin allerdings ein weiteres Problem an, das hier im folgenden nicht eingehender betrachtet werden kann, nämlich die Frage der Beziehung zwischen gesellschaftlichem politischem Selbstverständnis und politischer Praxis auf der einen, und theoretischer Wissenschaft und Philosophie auf der anderen Seite. Die angeführte Stelle scheint diesbezüglich für die voll artikulierte, demokratische Gesellschaft eine besondere, normativ ausgezeichnete Beziehung anzudeuten. 
und im „transzendenten“ Sinn hat. Voegelin greift hier zunächst einen Aspekt auf, der ihn schon in seinen Frühschriften, insbesondere in seinen Österreich-Studien beschäftigt hat und den er dort unter dem Thema Demokratie und Nation behandelt. Der Begriff der Nation bedeutet für Voegelin dort die im Vergleich zu traditionalen Gesellschaften besonders hohe Intensität bzw. „,Totalität“, in der Politik in modernen Gesellschaften auf der geistigen, politisch-kulturellen Durchdringung und Aktivierung einer Gesellschaft beruht. Nation bezeichnet die spezifisch moderne „,Durchdringung der Menschen eines Sozialzusammenhanges mit einer gemeinsamen Idee, einer gemeinsamen seelischen und geistigen Haltung, die Totalität der Weltanschauung.“ (1936: 23) Dieses Verständnis von Nation hat insofern unmittelbare Berührungspunkte mit der Demokratieproblematik, als es für Voegelin eine zentrale Voraussetzung demokratischer Politik beschreibt.

Die österreichische demokratische Verfassung von 1924 ist für Voegelin vor allem aus diesem Grund gescheitert: „Die Österreicher sind keine Nation“ (1930: 585); das österreichische Staatsproblem liegt im ,Nichtvorhandensein eines politischen Volkes, eines Demos, der den Staat als Demokratie hätte gründen können“ (1936: 93), schreibt Voegelin in den 30erJahren über die politische Entwicklung in Österreich. ${ }^{27}$ In der Neuen Wissenschaft betont Voegelin den gleichen Zusammenhang: Deskriptive Repräsentation setzt „existenziell““ einen Grad an Artikulierung der Gesellschaft voraus, wie er nur in modernen ,ppolitischen Nationen" erreicht wird. ${ }^{28}$

Die Formel vom voll artikulierten Individuum steht aber offenbar neben dieser ,existenziellen" Problematik auch für die eigentümlich demokratische Idee transzendenter Repräsentation, also für den spezifischen Legitimitäts- und Wahrheitsanspruch westlicher Demokratie. Die moderne „demokratische Symbolik“ ist, so Voegelin in der Neuen Wissenschaft, „die letzte Phase einer Reihe terminologischer Komplikationen, die im hohen Mittelalter zugleich mit der Artikulierung der westlichen politischen Gesellschaften beginnen“ (1959a: 63). In deren Verlauf entwickelt sich vor allem in der englischen Verfassungsgeschichte mit der schrittweisen Emanzipation und Trennung verschiedener Gewalten die Idee einer „eigenartige(n) vielschichtige(n) Repräsentanz“ (1959a: 64), deren zunächst nur angedeutete Implikationen sich schließlich im Selbstverständnis des westlichen Zivilregimes voll entfalten:

„Wenn die Artikulierung sich über die ganze Gesellschaft ausdehnt, dann wird auch der Repräsentant sich ausdehnen bis zu der Grenze, an der die Mitgliedschaft der Gesellschaft bis zum letzten Individuum politisch artikuliert

27 Eine ganz ähnliche Einschätzung der Situation Österreichs vor allem im 19. Jahrhundert findet sich übrigens auch bei Hannah Arendt: „Österreich-Ungarn ist nie ein Nationalstaat gewesen und hat das 19. Jahrhundert damit verbracht, die wesentliche Errungenschaft des Nationalstaats, die Verwandlung des aufgeklärten Despotismus in die konstitutionelle Monarchie und die Heranziehung einer unabhängigen Beamtenschaft, zu übernehmen, obwohl mit dem Fehlen einer homogenen Bevölkerung, der wichtigsten Vorbedingung für den Nationalstaat, auch jede Chance, sich wirklich zu modernisieren, [...] fortfiel.“ (Arendt 2006: 114)

28 Die Idee repräsentativer Institutionen im deskriptiven Sinn hat also die existenzielle „Artikulierung der Gesellschaft bis hinunter zum Individuum als vertretbarer Einheit zur Voraussetzung [...]. Dieser besondere Typus der Artikulierung ist nicht überall anzutreffen; er findet sich in der Tat nur in westlichen Gesellschaften." (Voegelin 1959a: 66) Voegelin verbindet hier den Nations- mit dem Demokratiebegriff in ganz ähnlicher Weise, wie das Will Kymlicka in seinen Studien zum Problem eines „liberalen Nationalismus“ tut. Vgl. z. B. Kymlicka 1999: 16-19. 
ist und dementsprechend die Gesellschaft ihr eigener Repräsentant wird. Symbolisch wird diese Grenze in der meisterhaften, dialektischen Prägnanz von Lincolns ,Regierung des Volkes, durch das Volk, für das Volk‘ erreicht. Das Symbol ,Volk' bedeutet in dieser Formel der Reihe nach die artikulierte politische Gesellschaft, ihren Repräsentanten und die Gesamtheit ihrer Glieder, die durch die Handlungen des Repräsentanten gebunden ist. Die unübertreffliche Fusion demokratischer Symbolik mit theoretischem Inhalt in dieser Formel ist das Geheimnis ihrer Wirksamkeit." (Voegelin 1959a: 66) ${ }^{29}$

Die „Artikulierung der Gesellschaft bis hinunter zum Individuum als vertretbarer Einheit" ist also nicht nur die existenzielle Voraussetzung demokratischer Institutionen, sondern sie impliziert in ihrer Konsequenz zugleich die normative Idee einer Regierung „des Volkes, durch das Volk, für das Volk“. Die volle Artikulierung der Gesellschaft mündet im westlichen Zivilregime in die politische Idee einer möglichst weitgehenden Identität von Repräsentant und Repräsentierten bzw. einer Rückbeziehung der politischen Formen von Repräsentation auf die Idee der Volkssouveränität im Sinne von ,popular government“ als der dem voll artikulierten Individuum adäquaten Form von Regierung. ${ }^{30}$ Moderne Demokratie als „westliches Zivilregime“ im normativen, „transzendenten“ Sinn bedeutet die politische Aktivierung und Durchdringung der Gesellschaft nicht mit irgendeiner Idee, sondern eben mit der Idee des voll artikulierten Individuums selbst.

\section{Eine „Philosophie der Demokratie“?}

Aus diesem ,individualistischen“ normativen Akzent der demokratischen Repräsentationsidee ergeben sich zwei entscheidende Implikationen für Voegelins Demokratieverständnis. Denn erstens hat Voegelin in diesem individualistischen bzw. personalistischen Akzent, wie bereits oben skizziert, schon in den 30er-Jahren den entscheidenden Unterschied zwischen zentraleuropäischer und angelsächsisch-westlicher Demokratie gesehen. Die existenzielle Artikulierung der modernen Gesellschaft und die zunehmende Interdependenz und sozioökonomische „Vermachtung“ der Beziehungen wird in den westlichen Zivilregimen konterkariert und ausbalanciert durch die Idee der „Dignität der Person“ bzw. „die freie Person des

29 Zwar kommt übrigens auch Kelsen auf diese zuletzt zitierten Stellen in der New Science zu sprechen, interpretiert sie jedoch lediglich als eine Inkonsequenz in Voegelins tendenziell eben doch anti-demokratischer Argumentation. (2004: 46).

30 Vgl. in diesem Zusammenhang Jürgen Gebhardts sehr treffende, sich an Voegelins Repräsentationstheorie anlehnende Charakterisierung des „neoklassisch-republikanischen“ Selbstverständnisses moderner westlicher Gesellschaften, wie es sich zuerst in der englischen und dann in der amerikanischen Revolution artikuliere: „In der englischen Revolution hatte der für die politische Kultur des Westens entscheidende Prozess der Selbstartikulation des politischen Individuums als Fundamentaldemokratisierung zu einer ersten Erschütterung der altständisch-monarchischen Ordnung geführt. Die naturrechtlich fundierte Idee des Individuums als letzte politische und spirituelle Einheit der Gesellschaft kündigte das demokratische Prinzip als das Konstituens politischer Ordnung an.“(2007: 20). 
Einzelnen“ (Voegelin 1935: 99 f.) und durch eine die Gleichheit aller Gesellschaftsmitglieder betonende Idee von Humanität (Voegelin 1932: 280 f.). ${ }^{31}$

Zweitens sieht Voegelin aber gerade in dieser Kernidee vom besonderen Status des Individuums, die sich in ihren politischen Implikationen erst im modernen „westlichen Zivilregime" voll entfaltet, dieses Zivilregime offenbar zugleich in einer letztlich ungebrochenen Kontinuität zur klassischen Tradition stehen. Denn die westliche Demokratie als Zivilregime findet ihr Fundament in einer republikanisch inspirierten aktiven Bürgerkultur, und sie wurzelt historisch in der , jüdisch-christliche[n] Vorstellung vom Menschen als Abbild Gottes, von seiner Würde, von seiner Unverletzlichkeit, [...] und zwar unabhängig von den Urteilen, welche die Gesellschaft über seinen Wert und über sein Betragen abgibt.“(Voegelin 1961: 55) ) $^{32}$

Aus ideengeschichtlicher Perspektive zeigt sich für Voegelin, dass der moderne Individualismus klassisch-republikanischen und christlichen Ursprungs ist bzw. - um es mit einer Weber'schen Metapher auszudrücken - dass der moderne Individualismus seine „Wurzeln“ in der klassisch-christlichen Idee von der „Dignität“ des Menschen als Person hat. Der moderne Individualismus und die klassische westliche Tradition politischen Denkens stehen in einem konstitutiven Verhältnis zueinander, sie sind durch eine fundamentale „Wahlverwandtschaft" miteinander verbunden. ${ }^{33}$ Max Webers Metaphorik aus der Protestantischen Ethik (1947: 83, 197, 202 ff.) passt auf Voegelins Perspektive nur bedingt, aber sie ist hier in der Tat sehr hilfreich, denn mit ihr lässt sich die eigentliche Pointe von Voegelins Perspektive in diesem Punkt besonders gut verdeutlichen. Es scheint, als komme es aus Voegelins vergleichender Perspektive auf das Problem moderner Demokratie gerade darauf an, dass diese „Wurzel“" des modernen Individualismus in der klassischen westlichen Tradition nicht „abstirbt“", dass die Idee des voll artikulierten Individuums sich in der Dynamik ihrer gesellschaftlichen Artikulierung nicht zum „Gespenst“ einer ehemals sinnvollen, substanziellen Idee verformt bzw. entleert, sondern, indem sie die politischen und philosophischen Implikationen des Personalismus der westlichen Tradition voll entfaltet und ausdifferenziert,

31 Dieser ausgeprägte Personalismus im Selbstverständnis der westlichen Demokratien sei unter anderem auch der Grund dafür, so Voegelins Einschätzung in den 30er-Jahren, dass die sowohl in Europa als auch in Amerika virulenten Rassenideen der Zeit im Gegensatz zu Zentraleuropa in den angelsächsischen Gesellschaften trotz aller faktischen Rassendiskriminierung letztlich dennoch nicht den Status sozialdominanter politischer Leitideen würden erreichen können (1935: 98 ff.).

32 Vgl. auch die folgende Stelle aus dem oben bereits zitierten Brief an Robert L. Fine, wo die „Kontinuität“ allerdings eher in dem oben skizzierten „negativen“ Sinne gedeutet wird, insbesondere in Voegelins Charakterisierung der spezifisch amerikanischen ,politischen Theologie“: Voegelin spricht dort von der „stabilization of the gnostic movement of Puritanism and Enlightenment at the level which may be called a secularized Independency. Obviously, in this respect some corrosion occurred before the foundation of the Republic. You can easily discern it in the political theology of the Declaration of Independence, with its significant shift from the early draft of ,sacred" truths to the gnostic final formula of ,self-evident" truths. But fortunately the ,self-evident" truths remained close to the not so self-evident Christian respect for the individual soul." (Voegelin 2007: 212)

33 Hier schließt sich u. a. die viel diskutierte Frage nach der Rolle der Religion für das Selbstverständnis moderner westlicher Gesellschaften im Allgemeinen und für den modernen Individualismus im Besonderen an. Vgl. dazu die zusammenfassenden Ausführungen zur „Genesis-“ und zur „Geltungshypothese“ bei Stein 2004: 323-328, die Beiträge in Walther (2004) und die These von der „Zivilreligion“ als „Liberalitätsgarant" bei Lübbe 1986: 210. 
zugleich das Bewusstsein von der Kontinuität zu dieser Tradition artikuliert und kritisch reflektiert.

Eine klassisch und republikanisch inspirierte und dennoch moderne Idee von der „Dignität des Einzelnen" auf der einen Seite, die Idee eines radikalen und gleichsam entwurzelten, anti-klassischen Individualismus auf der anderen Seite, in dem die Balance zwischen Kontinuität und Modernität verloren geht (Voegelin 1974: 20-28) und der in letzter Konsequenz in Kollektivismus und schließlich in den modernen Totalitarismus umschlagen kann: Das scheint mir für Voegelin einen, vielleicht den entscheidenden Unterschied zu bezeichnen zwischen ungehemmter und eingeschränkter Modernität - oder vielleicht könnte man es eben auch so verstehen: den Unterschied zwischen einer in einer komplexen, kritischen Kontinuität zur Tradition stehenden „mediterranen Modernität" einerseits (vgl. für diesen Begriff Voegelin 1998: 181 ff.) und einer ,gnostischen Modernität“", die den radikalen Bruch vollzieht, andererseits.

So formuliert impliziert das Problem der Moderne allerdings, dass die westliche Demokratie eben nicht als reiner Anachronismus bzw. als rein negatives, retardierendes Moment innerhalb der modernen Entwicklung, sondern mit Blick auf ihre genuine Modernität und ihren eigenständigen ,paradigmatischen Wert“ beschrieben werden müsste. Der historischempirischen Analyse und Beschreibung des Zivilregimes müsste dann auf der reflexiven Ebene so etwas wie eine „Theorie des Zivilregimes“ oder eine „Philosophie der Demokratie“ entsprechen, die sich etwa auf den „theoretischen Gehalt“" innerhalb der Lincoln'schen Formel konzentriert, von der Voegelin in der Neuen Wissenschaft spricht (siehe oben). ${ }^{34}$

Es ist bereits deutlich geworden, dass Voegelins Kritik der Moderne eine Theorie oder Philosophie der Demokratie in diesem Sinne keineswegs ausschließt. In einer Quelle aus dem Nachlass, einem schriftlich eingereichten Diskussionsbeitrag, der sich in Voegelins Arbeitsmaterial zur Jahrestagung der American Political Science Association von 1946 findet, kommt er auf die Frage einer solchen ,philosophy of democracy“ zu sprechen:

„In the course of this debate, a member of the Round Table has raised the complaint that we do not possess a ,philosophy of democracy“. The complaint is quite justified; but as long as it is raised in this general form, it has to remain without consequence for science. And if the complaint is not qualified, we may even run the risk of the misunderstanding that it is the function of political theory to justify a political system. The complaint would have to be reformulated in the following manner: that the theoretical penetration of the institutions and ideas of democracy leaves much to be desired, and that in recent years not much work has been done in this respect. Assuming these propositions to be valid, the scholar would have to decide whether he wishes to take up the problem of democracy at the point where Tocqueville left it in his study of American Democracy, or where J. St. Mill left it in his Essay on Liberty[...]“" (1946: 2 f.)

34 In einem Brief von 1970 beschreibt Voegelin Lincolns Formel vom ,government of the people, by the people, for the people“ in einem kritischeren Ton als eine ,transposition of a cosmological formula into a millenarian formula for a political action.“(1970: 1) Für eine Interpretation der Lincoln'schen Formel, die ihre kritische Kontinuität zur jüdisch-christlichen Tradition betont, vgl. auch Voegelin 1959b: 293 f. 
Voegelin nennt als weitere relevante Quellen Renan, LeBon, Karl Jaspers, Arnold Toynbee, Karl Mannheim und Harold Lasky. Die kurze Passage ist in verschiedener Hinsicht sehr interessant. Die Frage einer ,philosophy of democracy“ ist hier offenbar als ein wichtiges wissenschaftliches Desiderat angesprochen - unter der Einschränkung, dass sie nicht gleichsam als bloße Hagiografie der demokratischen Gesellschaft missverstanden werden dürfe. Und sie wird von Voegelin außerdem - das zumindest legen die genannten Anknüpfungspunkte nahe - als eine genuin moderne Fragestellung verstanden. Voegelin hat das skizzierte Programm wie gesagt nicht durchgeführt. Aber die oben dargestellten Überlegungen in der New Science geben die Richtung einer solchen theoretischen Behandlung der Demokratiethematik an. Nicht eine „reine Verfallsgeschichte“ und nicht die daraus sich ergebende notwendige Konsequenz des Leugnens der „politischen Errungenschaften“ der Neuzeit, sondern eine differenzierte Perspektive auf das Problem der Modernität und damit erstens die Kritik ihrer problematischen Aspekte und zweitens das oben skizzierte Programm einer „Theorie“ bzw. „Philosophie“ der Demokratie ist die plausible Entsprechung von Voegelins Beziehung zur Moderne, wie sie sich nach einem zweiten Blick in seine Schriften darstellt.

Und auch über die konkreteren Fragestellungen, die vor dem Hintergrund einer solchen Theorie zu diskutieren wären, lassen sich zumindest einige weitere Anhaltspunkte zusammentragen. Eine Reihe von Aufsätzen aus den 50er- und 60er-Jahren, in denen Voegelin auf die Demokratieproblematik zu sprechen kommt, ${ }^{35}$ thematisieren zum Beispiel einige solcher konkreteren Fragen, die sich für Voegelin mit dem Thema verbinden. Zentral sind dabei vor allem die egalitären und freiheitlichen Implikationen des Zivilregimes und deren politischkulturelle und sozioökonomische Bedingungen. Es seien erst die sozioökonomischen Verhältnisse der westlichen Moderne, wie Voegelin etwa in einem Aufsatz von 1959 betont, die eine politische Verwirklichung dieser Implikationen real möglich gemacht haben: Erst auf der Produktivitätsbasis der modernen Industriegesellschaft seien die Voraussetzungen des Zivilregimes, die noch Jefferson nur unter den Bedingungen der Agrarwirtschaft alten Stils für möglich gehalten habe, ,in den Bereich des Möglichen gerückt und zum Teil schon verwirklicht worden." (Voegelin 1959b: 299):

„Das Zivilregime ist [...] nur möglich in einer Gesellschaft freier unabhängiger Menschen. Und zur Freiheit des Menschen gehört eine materielle Basis, die ihm genügend Bewegungs-, Denk- und Bildungsfreiheit gewährt, um am öffentlichen Leben teilzunehmen - und v. a., um das öffentliche Leben als etwas zu erfahren, das ihn persönlich angeht.“(1959b: 299)

Voegelins konkrete institutionelle und politisch-kulturelle Überlegungen in diesem Zusammenhang konzentrieren sich auf die Frage, wie diese Möglichkeiten der Demokratie, die zugleich auch ihre Voraussetzungen sind, gegen die wachsende Interdependenz und die Vermachtungs- und Entfremdungstendenzen gesichert und offen gehalten werden können, die ebenfalls ein Signum der modernen westlichen Gesellschaften sind. Neben den freiheitlichen, egalitären und partizipatorischen Implikationen des Zivilregimes betont Voegelin dabei immer auch die Bedeutung von gesellschaftlichen Eliten gerade in modernen demokratischen Gesellschaften. Die zunehmende Interdependenz in komplexen Industriegesell-

35 Die im Original zum Teil nicht leicht zugänglichen Aufsätze sind in englischer Übersetzung im 11. Band der Collected Works veröffentlicht (Voegelin 2000). 
schaften zum Beispiel erfordere in zunehmendem Maße die Stärkung und die Schaffung neuartiger gesamtgesellschaftlich handlungsfähiger Akteure - Voegelin spricht in diesem Zusammenhang unter Anknüpfung an Ideen, die er schon in den 20er-Jahren bei John R. Commons kennengelernt hatte, von der „Demokratisierung der Unternehmerfunktion“ in der modernen Industriegesellschaft (Voegelin 1961, 1964). Er diskutiert hier einen Sachverhalt, den auch Tocqueville als entscheidendes Problem moderner Demokratie betrachtet hatte: das Fehlen bzw. den Verlust von in traditionell-aristokratischen Gesellschaften noch quasinatürlich vorgegebenen gesellschaftlichen Organisationsstrukturen und herausgehobenen Positionen, ohne die aber die Konstitution eines öffentlichen Raums und damit gesamtgesellschaftliche Kommunikation und Aktion nicht möglich seien. Tocqueville hat diesbezüglich die Bedeutung freier Assoziationen als des spezifisch demokratischen Ersatzes solcher vorgegebenen Strukturen betont (1984: 595-609). Voegelins Augenmerk liegt stärker auf der Rolle von kreativen Minderheiten und der mobilisierenden und organisierenden Wirkung herausgehobener Individuen. „[I]n der Tat ist die Struktur der Gesellschaft aus unbekannten Gründen hierarchisch und nicht egalitär; dazu wissen wir kein Mittel, um diesem Stand der Dinge Abhilfe zu schaffen“, so Voegelin etwa in einem Aufsatz von 1961 (55).

In diesem Punkt kommt neben der These von der faktischen Unumgänglichkeit gesellschaftlicher Eliten auch eine grundsätzlichere Skepsis gegenüber radikaldemokratischen Ideen zum Ausdruck - Ideen, die Voegelin in der Neuen Wissenschaft als ein typisches Charakteristikum „gnostischen“ Weltverständnisses beschrieben hat. ${ }^{36}$ Die klare Abgrenzung von diesen radikaldemokratischen, radikal-individualistischen und progressivistischen Vorstellungen bleibt ein charakteristischer Zug von Voegelins Demokratieverständnis. Darin spiegelt sich vor allem auch die Erfahrung der 30er- und 40er-Jahre und die vergleichende westlich-zentraleuropäische Perspektive, aus der Voegelin das Problem schon in seinen Frühschriften betrachtet und daher immer auch die Ambivalenzen und Probleme moderner Demokratisierungsprozesse betont hat. ${ }^{37}$ Moderne Demokratie hatte für Voegelin auch eine

36 Voegelin skizziert zum Beispiel die problematischen Implikationen der radikalen Idee einer hierarchie-, macht- und autoritätsfreien „Bruderschaft autonomer Personen“, die schon bei Joachim von Fiore entwickelt worden sei. (1959a: 160)

37 In der unmittelbaren Konfrontation mit dem Totalitarismus geriet ihm die Kritik solcher Tendenzen in einzelnen Bemerkungen auch zu einer Kritik der Demokratie schlechthin. In einem Brief an Hermann Broch aus dem Jahr 1945 zum Beispiel scheint sich Voegelin ganz auf eine altkonservative, elitistische Position zurückzuziehen, die im Prozess der Demokratisierung der modernen Gesellschaft selbst das eigentliche Problem der Zeit, gleichsam einen „Aufstand der Massen“ zu erkennen glaubt: „Ihre historischen Ausführungen implizieren ein Bild des demokratischen Problems, das sehr weit von meinem abweicht [...]. Sie nehmen z. B. an, dass die amerikanische Verfassung demokratisch sei. Das scheint mir geschichtlich zweifelhaft. Die Verfassungsbewegung ist nach 1786 (dem Jahr von Shay's Rebellion) in Fluss gekommen, als eine Maßnahme der Oligarchie, um die drohende Demokratie zu verhindern. Und der Charakter einer oligarchischen Organisation des ancien regime ist erhalten geblieben, trotz der Demokratisierung des Wahlrechts. Im oligarchischen Charakter der amerikanischen Gesellschaft würde ich gerade ihre Widerstandskraft gegen den sogenannten „Fascismus“ sehen. Während ich den Faschismus deutscher Prägung als eine echt demokratische Bewegung klassifizieren würde, welche nicht mehr durch die Standards des ancien regime gehemmt ist. [...] Wenn Hitler und Mussolini die wahre Demokratie für sich in Anspruch nehmen, so haben sie, fürchte ich, recht.“(Voegelin 1945). 
„totalitäre“ Variante; der Totalitarismus erschien ihm in diesem Sinne nicht als den modernen Demokratisierungsprozessen gegenüberstehendes, fremdes Phänomen, sondern als eine diesen Prozessen selbst inhärente Gefahr. ${ }^{38}$

Dessen ungeachtet ist die volle „Artikulierung“, d. h. eine Demokratisierung der Gesellschaft im „existenziellen“ und im ,transzendenten“ Sinn für ein demokratisches Zivilregime für Voegelin die unabdingbare Voraussetzung. Im Fehlen dieser Voraussetzung hat Voegelin noch in den 60er-Jahren das entscheidende Problem der deutschen Nachkriegsdemokratie gesehen. In einem Brief an Kurt Sontheimer vom Oktober 1966 schreibt Voegelin zum Problem eines erstarkenden anti-demokratischen Nationalismus in Deutschland: „Ich halte die nationalistische Gefahr für größer als sie an der Oberfläche der vorläufig unbedeutenden Phänomene erscheint, weil eben wegen des Fehlens einer demokratischen, revolutionären Tradition der Widerstand gegen den Nationalismus durch die sogenannten Demokraten nicht die genügende Stärke entfalten kann. Irgendwann wird doch noch die demokratische Revolution in Deutschland stattfinden müssen." $(1966)^{39}$

\section{Schlussfolgerungen}

Vor dem Hintergrund von Voegelins Überlegungen zum westlichen Zivilregime ergibt sich ein ambivalenteres, differenzierteres Bild der Neuzeit und Moderne, als es seine zum Teil bewusst überspitzten Krisendiagnosen zunächst nahelegen. Neben die Beschreibung der gnostischen Moderne als eines radikalen Bruchs und als Verfallsprozess tritt die Vorstellung einer in Kontinuität zur klassischen Tradition stehenden kulturellen und politischen Entwicklung, die aber ihrerseits genuin moderne Züge trägt. Der Topos „,westliches Zivilregime“ impliziert vor diesem Hintergrund bei Voegelin nicht so sehr die These von einem einfachen Gegensatz von Klassik und Neuzeit, von Alt versus Neu, sondern vielmehr die Frage einer Balance innerhalb der Neuzeit selbst zwischen Kontinuität einerseits und Modernität und Differenzierung andererseits. Der Verlust dieser Balance bzw. der Verlust der Kontinuität ist das Problem des modernen Gnostizismus, der, wie Voegelin in der New Science schreibt, in dem Bestreben besteht, „die Wahrheit der Seele nicht zu ergänzen, sondern zu ersetzen.“ (1959a: 225) Dem gegenüber steht das westliche Zivilregime exemplarisch für eine in Kontinuität zur Klassik stehende „Zivilisationsströmung“, die aber dennoch genuin modern ist. Eine philosophische Interpretation moderner Demokratie in diesem Sinne impliziert nicht nur ein affirmatives Verhältnis zu den zentralen politischen „Errungenschaften der Neuzeit“. Sondern sie impliziert darüber hinaus bei Voegelin die These von der substanziellen Kontinuität westlichen politischen Denkens gerade im Hinblick auf diese Errungenschaften, allerdings einer Kontinuität, die in einem Prozess der Entwicklung und Differenzierung Ausdruck findet. Sie impliziert ein im Wortsinn ,neoklassisches“ Verständnis von Moderne

38 Vgl. zum Konzept einer ,totalitären Demokratie“ auch die klassische Studie von J. L. Talmon 1961.

39 Voegelins Brief bezieht sich auf einen Aufsatz, in dem sich Kurt Sontheimer (1966) kritisch mit der bundesdeutschen Situation der Zeit auseinandersetzt und unter Bezugnahme auf die konservativen Parteien und Teile der Jugendbewegung und des intellektuellen Diskurses auf problematische „nationalistische“ Tendenzen in der öffentlichen Debatte aufmerksam macht. 
als einer politisch und theoretisch eigenständigen Phase eines sich differenzierenden und dabei immer auch kritisch selbst reflektierenden Prozesses westlichen politischen Denkens.

Eine solche Perspektive hat Voegelin an verschiedenen Stellen seines Werks angedeutet und in einigen ihrer wichtigsten Implikationen diskutiert, allerdings nicht ausgearbeitet und systematisch entwickelt. Eine Abhandlung zum Thema „Wissenschaft, Politik und westliches Zivilregime“, sozusagen das Gegenstück zu seiner bewusst provozierenden Münchner Antrittsvorlesung „Wissenschaft, Politik und Gnosis“ fehlt in Voegelins Oeuvre. Dieses Fehlen - will man dann doch den Versuch wagen, ein nicht geschriebenes Buch zu interpretieren - ist aber keineswegs als die unausweichliche Konsequenz einer wissenschaftlichen Konzeption zu verstehen, die auf dem Narrativ einer „reinen Verfallsgeschichte“ basiert. Vor dem Hintergrund der skizzierten Überlegungen Voegelins zum westlichen Zivilregime erscheint eine andere Deutung plausibler: Dieses Fehlen ist eher als eine konzeptionell nicht nur keineswegs notwendige, sondern darüber hinaus als eine „Lücke“ zu verstehen, der an zahlreichen Stellen des Oeuvres Andeutungen, Anknüpfungspunkte und aufgeworfene, zum Teil offen gebliebene Fragen entsprechen - eine Lücke also, die in diesem Sinne in der Tat gewisse „Phantomschmerzen“ im Werk hinterlassen hat.

Vielleicht zeigt sich darin auch eine beschränkende Wirkung, welche die Erfahrung des Totalitarismus auf Voegelins wissenschaftliche Perspektive hatte. Das Problem der „Krise“, die Kritik der problematischen Aspekte des modernen Selbstverständnisses, zuweilen auch der bewusst polemisch geführte intellektuelle Kampf gegen die totalitären Massenideologien - das sind vor dem Hintergrund der Ereignisse seiner Zeit die Themen, die Voegelins Denken vor allem in den 50er- und 60er-Jahren klar dominieren. ${ }^{40}$ Dessen ungeachtet aber - bzw. gerade dann, wenn man in diesem Punkt eine gewisse zeitbedingte Beschränkung von Voegelins Perspektive erkennen mag - kann eine Interpretation von Voegelins Werk, die sich ihrerseits auf diese zeitweise Dominanz der Krisenproblematik beschränkte, nur ein reduziertes Bild seiner politischen Wissenschaft liefern. Die hier entwickelte Rekonstruktion von Voegelins Deutung des westlichen Zivilregimes hat versucht zu zeigen, dass seine neoklassische Perspektive über die Krisendiagnose hinaus gehende und äußerst interessante Beiträge zur für die politische Theorie weiterhin aktuellen Debatte um das Problem der Moderne und das Selbstverständnis moderner westlicher Gesellschaften leisten kann. Unter anderem kann sie dazu beitragen, die gängige Semantik des Verlusts, des radikalen Bruchs und des „heute nicht mehr" - die nicht nur den aktuellen Krisendiskurs, sondern ebenso deutlich einen Großteil desjenigen theoretischen Diskurses prägt, der bewusst eine affirmative Position zur Moderne einnimmt - durch eine Semantik des ,,heute in anderer Weise“, d. h. eine Semantik der Differenzierung und komplexen Kontinuität zu ergänzen und zum Teil auch kritisch zu hinterfragen.

40 Diese Dominanz, die Konzentration auf die „Krise“ und vielleicht auch die Bereitschaft zur Polemik und zum intellektuellen Widerstand empfand Voegelin offenbar vor allem als moralische Forderung, die seine Zeit an den politischen Denker stellte. Vgl. dazu das aufschlussreiche Motto, ein Zitat von Richard Hooker, das Voegelin der Neuen Wissenschaft vorangestellt hat: „Posterity may know we have not loosely through silence permitted things to pass away as in a dream." 
Krise der Moderne und moderne Demokratie

\section{Literatur}

Arendt, Hannah, 2006: Elemente und Ursprünge totaler Herrschaft, München/Zürich (11. Aufl.).

Barley, Delbert, 1990: Hannah Arendt. Einführung in ihr Werk, Freiburg/München.

Barnouw, Dagmar, 2003: Autorität und Freiheit: Hannah Arendt und die politische Modernität Amerikas, in: Thaa/Probst 2003, 47-68.

Benhabib, Seyla, 1996: The Reluctant Modernism of Hannah Arendt, Thousand Oaks.

Bluhm, Harald, 2003: Von Weimarer Existentialphilosophie zum politischen Denken. Hannah Arendts Krisenkonzept und ihre Auffassung politischer Erfahrung, in: Thaa/Probst 2003, 69-92.

Faber, Richard, 1984: Der Prometheus-Komplex. Zur Kritik der Politotheologie Eric Voegelins und Hans Blumenbergs, Würzburg.

Gebhardt, Jürgen, 1989: Eric Voegelin und die neuere Entwicklung der Geisteswissenschaften, in: Zeitschrift für Politik 36, 251-263.

Gebhardt, Jürgen, 1995: Zwischen Wissenschaft und Religion. Zur intellektuellen Biographie Eric Voegelins in den 30er Jahren, in: Politisches Denken, Jahrbuch, 283-304.

Gebhardt, Jürgen, 2004: Politische Kultur und Zivilreligion, in: ders., Politik, Hermeneutik, Humanität. Gesammelte Aufsätze, hrsg. von Clemens Kauffmann/Helmut Klumpjan/ Matthias Riedl/Hans-Jörg Sigwart, Berlin, 101-116.

Gebhardt, Jürgen, 2007: Idee und Wirklichkeit der „Representative Republic“, in: Winfried Thaa (Hrsg.), Inklusion durch Repräsentation, Baden-Baden, 19-37.

Geisen, Thomas, 2003: Hannah Arendt und Kritische Theorie Horkheimers und Adornos. Zum Verhältnis von soziologischem und politischem Denken, in: Thaa/Probst 2003, 93-115.

Gutschker, Thomas, 2002: Aristotelische Diskurse. Aristoteles in der politischen Philosophie des 20. Jahrhunderts, Stuttgart/Weimar.

Henkel, Michael, 2003: Positivismuskritik und autoritärer Staat. Die Grundlagendebatte in der Weimarer Staatsrechtslehre und Eric Voegelins Weg zu einer neuen Wissenschaft der Politik (bis 1938), München (Occasional Papers XXXVI, Eric-Voegelin-Archiv, Ludwig-Maximillians-Universität).

Henkel, Michael, 2008: Eine Suche nach Ordnung in der Geschichte der Menschheit. Über Eric Voegelins Schriften in deutscher Sprache, in: ZPol 18, 257-282.

Henningsen, Manfred, 2000: Editor's Introduction, in: The Collected Works of Eric Voegelin, Vol. 5: Modernity Without Restraint. Edited with an Introduction by Manfred Henningsen, Columbia/London, 1-17.

Hollweck, Thomas, 1999: Das gnostische Wesen der Moderne: Die Zukunft einer These, in: Eric Voegelin, Der Gottesmord. Zur Genese und Gestalt der modernen politischen Gnosis. Hrsg. und eingeleitet von Peter J. Opitz; mit einem Nachwort von Thomas Hollweck, München, 144-159.

Kauffmann, Clemens, 2000: Strauss und Rawls. Das philosophische Dilemma der Politik, Berlin. 
Kelsen, Hans, 2004: A New Science of Politics. Hans Kelsen's Reply to Eric Voegelin's „New Science of Politics“. A Contribution to the Critique of Ideology. Edited by Eckhart Arnold, Frankfurt/Lancaster.

Kiel, Albrecht, 1998: Gottesstaat und Pax Americana. Zur politischen Theologie von Carl Schmitt und Eric Voegelin, Cuxhaven/Dartford.

Kymlicka, Will, 1999: Multikulturalismus und Demokratie. Über Minderheiten in Staaten und Nationen, Hamburg.

Leutzsch, Martin, 1994: Der Bezug auf die Bibel und ihre Wirkungsgeschichte bei Carl Schmitt, in: Bernd Wacker (Hrsg.), Die eigentlich katholische Verschärfung ... Konfession, Theologie und Politik im Werk Carl Schmitts, München, 175-202.

Llanque, Marcus, 1995: Die Theorie politischer Einheitsbildung in Weimar und die Logik von Einheit und Vielheit (Rudolf Smend, Carl Schmitt, Hermann Heller), in: Andreas Göbel/Dirk van Laak,/Ingeborg Villinger (Hrsg.), Metamorphosen des Politischen. Grundfragen politischer Einheitsbildung seit den 20er Jahren, Berlin, 157-176.

Lübbe, Hermann, 1986: Staat und Zivilreligion. Ein Aspekt politischer Legitimität, in: Heinz Kleger/Alois Müller (Hrsg.), Religion des Bürgers. Zivilreligion in Amerika und Europa, München, 195-220.

Maier, Hans (Hrsg.), 1996 ff.: Totalitarismus und Politische Religionen. Konzepte des Diktaturvergleichs, 3 Bde., Paderborn.

McAllister, Ted V., 1996: Revolt against Modernity. Leo Strauss, Eric Voegelin, and the Search for a Postliberal Order, Kansas.

Menke, Christoph/Pollmann, Arnd, 2007: Philosophie der Menschenrechte zur Einführung, Hamburg.

Noetzel, Thomas, 1991: Eric Voegelin: Episteme und Doxai - Eine Verfallsgeschichte des politischen Wissens, in: Hans Karl Rupp/Thomas Noetzel (Hrsg.), Macht, Freiheit, Demokratie: Anfänge der westdeutschen Politikwissenschaft, Marburg, 137-152.

Opitz, Peter J., 1989: Spurensuche - Zum Einfluss Eric Voegelins auf die Politische Wissenschaft in der Bundesrepublik Deutschland, in: Zeitschrift für Politik 36, 235-250.

Opitz, Peter J., 1999: Die Gnosis-These - Anmerkungen zu Eric Voegelins Interpretation der westlichen Moderne, in: Eric Voegelin, Der Gottesmord. Zur Genese und Gestalt der modernen politischen Gnosis. Hrsg. und eingeleitet von Peter J. Opitz; mit einem Nachwort von Thomas Hollweck, München, 7-35.

Ottmann, Henning, 2005: Platon, Aristoteles und die neoklassische Philosophie der Gegenwart, Baden-Baden.

Ottmann, Henning, 2006: Geschichte des politischen Denkens. Band 3: Die Neuzeit. Teilband 1: Von Machiavelli bis zu den großen Revolutionen, Stuttgart/Weimar.

Pitkin, Hanna Fenichel, 1967: The Concept of Representation, Berkeley/Los Angeles.

Sandoz, Ellis, 2000: The Voegelinian Revolution. A Biographical Introduction, New Brunswick/London (2. Aufl.).

Schaal, Gary S., 2006: Zwischenbetrachtung: Entwicklungspfade der Politischen Theorie nach 1945, in: André Brodocz/Gary S. Schaal (Hrsg.), Politische Theorien der Gegenwart. Eine Einführung, Opladen/Farmington Hills (2., erweiterte und aktualisierte Auflage), 499-539. 
Schwelling, Birgit, 2005: Kulturwissenschaftliche Traditionslinien in der Politikwissenschaft: Eric Voegelin revisited, in: Zeitschrift für Politik 52, 3-24.

Sigwart, Hans-Jörg, 2005: Das Politische und die Wissenschaft. Intellektuell-biographische Studien zum Frühwerk Eric Voegelins, Würzburg.

Sigwart, Hans-Jörg, 2007a: Eine Hermeneutik des Politischen. Eric Voegelins Entwurf einer geisteswissenschaftlichen Staats- und Herrschaftslehre, in: Peter J. Opitz (Hrsg.), Erich Voegelins Herrschaftslehre: Annäherungen an einen schwierigen Text, München (Occasional Papers LVII, Eric-Voegelin-Archiv, Ludwig-Maximillians-Universität), 19-39.

Sigwart, Hans-Jörg, 2007b: Wirklichkeitswissenschaft und Ordnungswissenschaft: Eric Voegelins Auseinandersetzung mit Max Weber, in: Zeitschrift für Politik 54, 379-407.

Skinner, Quentin, 1988: Machiavelli zur Einführung, Hamburg.

Söllner, Alfons, 1996: Deutsche Politikwissenschaftler in der Emigration. Studien zu ihrer Akkulturation und Wirkungsgeschichte, Opladen.

Sontheimer, Kurt, 1966: Die Wiederkehr des Nationalismus in der Bundesrepublik, in: Tribüne 5 (18), 1916-1934.

Stein, Tine, 2004: Religion, in: Gerhard Göhler/Mattias Iser/Ina Kerner (Hrsg.), Politische Theorie. 22 umkämpfte Begriffe zur Einführung, Wiesbaden, 315-331.

Talmon, J. L., 1961: Die Ursprünge der totalitären Demokratie, Köln/Opladen.

Thaa, Winfried, 2005: Kulturkritik und Demokratie bei Max Weber und Hannah Arendt, in: Zeitschrift für Politik 52, 25-56.

Thaa, Winfried/Probst, Lothar (Hrsg.), 2003: Die Entdeckung der Freiheit. Amerika im Denken Hannah Arendts, Berlin/Wien.

Tocqueville, Alexis de, 1984: Über die Demokratie in Amerika. Hrsg. von Jacob P. Mayer, München (2. Aufl.).

Voegelin, Eric, 1928: Über die Form des amerikanischen Geistes, Tübingen.

Voegelin, Eric, 1930: Die österreichische Verfassungsreform von 1929, in: Zeitschrift für Politik XIX (9), 585-615.

Voegelin, Eric, 1932: Nachwort, in: Ernst Dimnet, Die Kunst des Denkens, Freiburg, 279-296.

Voegelin, Eric, 1935: Rasse und Staat, in: Otto Klemm (Hrsg.), Psychologie und Gemeinschaftsleben, Jena, 91-104.

Voegelin, Eric, 1936: Der autoritäre Staat. Ein Versuch über das österreichische Staatsproblem, Wien.

Voegelin, Eric, 1939: „Democracy and the Individual.“ Unveröffentlichtes Manuskript, in: Eric Voegelin Archive, Hoover Institution, Stanford University, Box 56, Folder 4.

Voegelin, Eric, 1945: Brief an Hermann Broch, 10. September 1945, in: Eric Voegelin Archive, Hoover Institution, Stanford University, Box 8, Folder 42.

Voegelin, Eric, 1946: „Remarks in Discussion“, in: Eric Voegelin Archive, Hoover Institution, Stanford University, Box 62, Folder 16.

Voegelin, Eric, 1959a: Die neue Wissenschaft der Politik, München. (Neuste Ausgabe 2007: Die neue Wissenschaft der Politik. Eine Einführung, hrsg. von Peter J. Opitz, mit einem Nachwort des Herausgebers und einer Bibliographie, Paderborn/München.)

Voegelin, Eric, 1959b: Demokratie im neuen Europa, in: Gesellschaft - Staat - Erziehung, Heft 7, 293-300. 
Voegelin, Eric, 1959c: Wissenschaft, Politik und Gnosis, München.

Voegelin, Eric, 1960: Religionsersatz. Die gnostischen Massenbewegungen unserer Zeit, in: Wort und Wahrheit 15, 5-18.

Voegelin, Eric, 1961: Die industrielle Gesellschaft auf der Suche nach der Vernunft, in: Raymond Aron u. a., Die industrielle Gesellschaft und die drei Welten. Das Seminar von Rheinfelden, Zürich, 46-64.

Voegelin, Eric, 1964: Demokratie und Industriegesellschaft, in: Veröffentlichungen der Walter-Raymond-Stiftung, Bd. 4, Köln, 92-114.

Voegelin, Eric, 1966: Brief an Kurt Sontheimer, 31. Oktober 1966, in: Eric Voegelin Archive, Hoover Institution, Stanford University, Box 13, Folder 8.

Voegelin, Eric, 1970: Brief an Mel Bradford, 6. Oktober 1970, in: Eric Voegelin Archive, Hoover Institution, Stanford University, Box 8, Folder 34.

Voegelin, Eric, 1974: Order and History, Vol. 4: The Ecumenic Age, Baton Rouge/London. Voegelin, Eric, 1987: The New Science of Politics, Chicago/London (2. Aufl.).

Voegelin, Eric, 1989: Autobiographical Reflections. Edited with an Introduction by Ellis Sandoz, Baton Rouge/London (deutsche Übersetzung 1994: Autobiographische Reflexionen, München)

Voegelin, Eric, 1998: History of Political Ideas V: Religion and the Rise of Modernity. The Collected Works of Eric Voegelin, Vol. 23. Edited with an Introduction by James L. Wiser, Columbia/London.

Voegelin, Eric, 1999: Der Gottesmord. Zur Genese und Gestalt der modernen politischen Gnosis. Hrsg. und eingeleitet von Peter J. Opitz; mit einem Nachwort von Thomas Hollweck, München.

Voegelin, Eric, 2000: Published Essays 1953-1965. The Collected Works of Eric Voegelin, Vol. 11. Edited with an Introduction by Ellis Sandoz, Columbia/London.

Voegelin, Eric, 2001 ff.: Ordnung und Geschichte, 10 Bde., hrsg. von Peter J. Opitz und Dietmar Herz, München.

Voegelin, Eric, 2005: Anamnesis. Zur Theorie der Geschichte und Politik, Freiburg/München (zuerst 1966).

Voegelin, Eric, 2006: Hitler und die Deutschen, hrsg. von Manfred Henningsen, München.

Voegelin, Eric, 2007: Brief an Arthur L. Fine, 12. Februar 1954, in: Selected Correspondence 1950-1985. The Collected Works of Eric Voegelin, Vol. 30. Edited with an Introduction by Thomas Hollweck, Columbia/London, 212-214.

Voegelin, Eric/Schütz, Alfred/Strauss, Leo/Gurwitsch, Aron, 1993: Briefwechsel über „Die Neue Wissenschaft der Politik“. Hrsg. von Peter J. Opitz, Freiburg/München.

Walther, Manfred (Hrsg.), 2004: Religion und Politik. Zu Theorie und Praxis des theologisch-politischen Komplexes, Baden-Baden.

Weber, Max, 1947: Die protestantische Ethik und der Geist des Kapitalismus, in: ders., Gesammelte Aufsätze zur Religionssoziologie, Bd. 1, Tübingen, 16-206.

Weiss, Gilbert, 2000: Theorie, Relevanz und Wahrheit. Eine Rekonstruktion des Briefwechsels zwischen Eric Voegelin und Alfred Schütz (1938-1959), München. 
Korrespondenzanschrift:

Dr. Hans-Jörg Sigwart

Institut für Politische Wissenschaft

Friedrich-Alexander-Universität Erlangen-Nürnberg

Kochstraße 4

91052 Erlangen

E-Mail: hgsigwar@phil.uni-erlangen.de

Web: http://www.polwis.phil.uni-erlangen.de/

\section{UN - Die Weltorganisation}

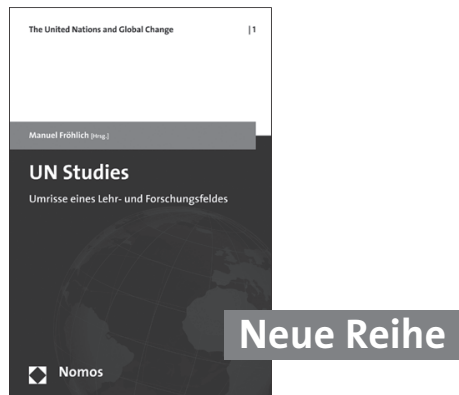

\section{UN Studies}

Umrisse eines Lehrund Forschungsfeldes

Herausgegeben von

Prof. Dr. Manuel Fröhlich 2008, 269 S., brosch., 29,90€, ISBN 978-3-8329-3452-1 (The United Nations and Global Change, Bd. 1)

Bitte bestellen Sie im Buchhandel oder

versandkostenfrei unter www.nomos-shop.de

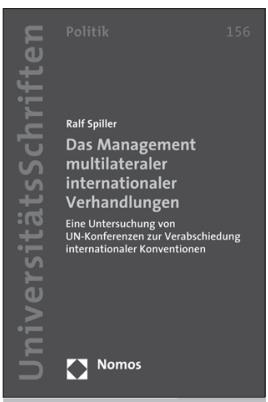

\section{Das Management multilateraler} internationaler Verhandlungen

Eine Untersuchung von

UN-Konferenzen zur Verabschiedung internationaler Konventionen

Von Dr. Ralf Spiller 2008, 296 S., brosch., 49,-€, ISBN 978-3-8329-3616-7

(Nomos Universitätsschriften Politik, Bd. 156)

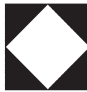

